\title{
1 Synapse-associated astrocyte mitochondria stabilize motor circuits to prevent
}

2 excitotoxicity

4 Sonja A. Zolnoski ${ }^{1}$, Emily L. Heckman ${ }^{1}$, Chris Q. Doe $^{1}$, and Sarah D. Ackerman ${ }^{1,2 *}$

$7{ }^{1}$ Institute of Neuroscience, Howard Hughes Medical Institute, University of Oregon, Eugene,

$8 \quad$ OR 97403

$9 \quad{ }^{2}$ Current address: Center for Brain Immunology and Glia, Department of Pathology and

10 Immunology, Washington University School of Medicine, Saint Louis, MO 63110

$13 *$ Author for correspondence at sarah.ackerman@ wustl.edu (SDA)

Orcid IDs: 0000-0002-0012-3364 (ELH), 0000-0001-5980-8029 (CQD), 0000-0001-8752-8972 (SDA) 


\section{ABSTRACT}

Early stages of the devastating neurodegenerative disease amyotrophic lateral sclerosis (ALS) are characterized by motor neuron hyperexcitability. During this phase, peri-synaptic astrocytes are neuroprotective. When reactive, loss of wild-type astrocyte functions results in excitotoxicity. How astrocytes stabilize motor circuit function in early-stage ALS is poorly understood. Here, we used Drosophila motor neurons to define the role of astrocyte-motor neuron metabolic coupling in a model of ALS: astrocyte knockdown of the ALS-causing gene tbph/TARDBP. In wild-type, astrocyte mitochondria were dynamically trafficked towards active motor dendrites/synapses to meet local metabolic demand. Knockdown of tbph in astrocytes resulted in motor neuron hyperexcitability, reminiscent of early-stage ALS, which was met with a compensatory accumulation of astrocyte mitochondria near motor dendrites/synapses. Finally, we blocked mitochondria-synapse association in tbph knockdown animals and observed locomotor deficits and synapse loss. Thus, synapseassociated astrocyte mitochondria stabilize motor circuits to prevent the transition from hyperexcitability to excitotoxicity. 


\section{MAIN}

Amyotrophic lateral sclerosis (ALS) is devastating neurodegenerative disorder in which motor neurons are selectively lost from the central nervous system (CNS), resulting in paralysis and eventual death. One major hurdle to understanding the molecular and cellular basis of ALS is the genetic complexity of the disease, which can be caused or modified by hundreds of mutations in greater than 50 genes $^{1-4}$. Furthermore, less than ten percent of cases with a known genetic basis are hereditary (familial ALS); thus, each mutation only represents a very small percentage of the overall population suffering from $\mathrm{ALS}^{1}$. For example, mutations in TAR DNA-binding protein 43 (TDP-43, encoded by the gene TARDBP) represent $1.5-4.2 \%$ of familial ALS, but only .2-.8\% of all ALS cases ${ }^{5}$. Despite the low representation of TARDBP mutations in the overall ALS population, changes in TDP-43 localization are apparent in ninety-five percent of ALS and forty-five percent of overlapping frontotemporal dementia (FTD) cases ${ }^{6}$. Thus, TDP-43 represents a promising therapeutic target to tackle the pathophysiological heterogeneity of ALS/FTD.

TDP-43 is a nuclear DNA/RNA binding protein that broadly regulates RNA metabolism and subsequent gene expression. In ALS/FTD neurons and associated glia, TDP43 is translocated from the nucleus to form dense cytoplasmic inclusions. Accordingly, it is thought that TDP-43-associated neuronal death results from both loss-of-function and toxic gain-of-function phenotypes ${ }^{7,8}$. Although ALS is considered a motor neuron disease, recent reports suggest that glial dysfunction can strongly modulate disease progression ${ }^{9-12}$. Astrocytes -the most abundant glial subtype- extend finely branched processes to intimately ensheath central synapses, including those at motor neuron dendrites. This unique positioning allows astrocytes to play several key roles that ensure neuronal health: provision of metabolic 
support, ion buffering, neurotransmitter recycling, and regulation of synaptic

structure/function ${ }^{13}$. In ALS, healthy astrocytes undergo dramatic changes in cell morphology and gene expression profile to transform from a functional homeostatic to dysfunctional reactive state ${ }^{1,14,15}$. The early phases of ALS are characterized by motor neuron hyperexcitability ${ }^{16}$, and transformation of astrocytes into a reactive state is thought to tip the balance from motor neuron hyperexcitability to excitotoxicity ${ }^{16,17}$. In contrast, the presence of wild-type astrocytes in an otherwise mutant background is sufficient to slow disease onset and progression in murine models of ALS ${ }^{18}$. Thus, discovering which wild-type function(s) of astrocytes are most neuroprotective in pre-symptomatic ALS is of great clinical importance. We took advantage of Drosophila as a system with well-established ALS models and precise genetic access to motor neurons and astrocytes ${ }^{19,20}$. Specifically, we wanted to test the hypothesis that reduction in astrocyte-motor neuron metabolic coupling transforms motor circuit activity from hyperactive to excitotoxic in an ALS model. In the healthy nervous system, we found that astrocytes position mitochondria near motor dendrites/synapses in an activity-dependent manner, presumably to match metabolic demand. Following astrocytespecific knockdown of tbph (human TARDBP), we observed increased motor neuron activity and increased localization of astrocyte mitochondria near motor dendrites/synapses, but no excitotoxicity or behavioral changes. These data suggest that astrocyte mitochondria are tuned to neighboring neuronal activity, and that proximity to synapses may be neuroprotective in conditions where motor activity is elevated (e.g. astrocyte knockdown of $t b p h$ ). To test this hypothesis, we blocked mitochondrial trafficking in tbph knockdown animals and assessed the effect on motor circuit structure and function. When mitochondria cannot form stable associations with synapses in tbph knockdown animals, we observed significant increases in 
crawling speed, consistent with motor neuron hyperexcitability, which preceded synapse loss at the neuromuscular junction, consistent with excitotoxicity. We conclude that astrocytes dynamically position their mitochondria to match local metabolic demand and facilitate longterm stability of motor circuits.

\section{RESULTS}

\section{Activity-dependent positioning of astrocyte mitochondria}

Astrocytes are an essential energy source for neurons, facilitating both neuronal longevity and long-term memory formation ${ }^{13,21,22}$. Not surprisingly, mitochondria are frequently visualized inside fine astrocytic peri-synaptic processes by transmission electron microscopy ${ }^{23}$. We sought to test whether ALS-causing mutations resulted in defective astrocyte-neuron metabolic coupling and excitotoxicity. As diseased motor neurons exhibit elevated activity in mammals ${ }^{1}$, we first needed test if astrocyte mitochondria localization correlates with motor neuron activity. We focused our work on the well-characterized aCC/RP2 motor neurons of the Drosophila larval motor circuit ${ }^{24}$. We developed an image analysis pipeline in Imaris to define presynapses that were co-localized with aCC/RP2 dendrites, similar to one previously published $^{20,25}$. We then determined the percentage of these synapses that were associated with astrocyte mitochondria (Figure 1, see legend and methods for full details). Using these model neurons, we recently discovered a critical period of motor circuit plasticity during development when motor neurons robustly change their dendrite structure in response to neuronal activity ${ }^{20}$. To assess activity-dependent mitochondria-synapse association without the confounds of structural plasticity, we limited our studies to larval stages $\geq 8$ hours after larval hatching (h ALH), when the critical period is closed and dendrite morphology is not 
dramatically affected by motor neuron activity. Following optogenetic activation of motor neurons for one hour (RN2-lexA, lexAop-Chrimson::tdTomato), we observed a redistribution of astrocyte mitochondria (R25H07-gal4, UAS-mito::GFP) from the periphery to the center of the dendritic domain (Figure 2a-b"). This resulted in a significant increase in total astrocyte mitochondrial volume within the dendritic domain, and a significant increase in the percentage of motor synapses within close proximity $(\leq 120 \mathrm{~nm})$ to astrocyte mitochondria (Figure 2c-d). Importantly, we did not observe any activity-dependent changes in overall dendrite volume that could account for these phenotypes, as the critical period was closed (Figure 2e).

Previous mammalian slice culture assays demonstrated that acute, pharmacological activation of neurons results in pausing of astrocyte mitochondria near synapses in a calciumdependent manner ${ }^{26,27}$. To determine if Drosophila motor neuron activity causes astrocyte mitochondrial stalling at motor dendrites/synapses, we used live imaging to visualize realtime changes in mitochondrial behavior during optogenetic activation of neighboring motor neurons. In contrast to simple stalling of mitochondria at synapses, we observed rapid, active transport of astrocyte mitochondria from the periphery to the center of the dendritic domain within one minute of motor neuron activation, resulting in a significant increase in mitochondrial volume within the activated region. Interestingly, this volume plateaued after five minutes of stimulation, suggesting that in vivo, mitochondria are initially actively transported to dendrites/synapses, followed by pausing once a certain threshold of activity has passed (Figure 2f-g, Supplemental Movies 1-2).

We next tested whether optogenetic silencing of motor neurons (RN2-gal4, UASGtACR2::EYFP) alters astrocyte mitochondrial distribution (alrm-lexA, lexAop- 
mcherry::mito.OMM, Figure 3). Interestingly, while motor neuron activation was sufficient to recruit astrocyte mitochondria to synapses, synapse-associated mitochondria were largely stable throughout motor neuron silencing (Figure 3a-c). We did, however, observe a significant decrease in overall mitochondrial volume within the dendritic domain after silencing for one hour (Figure 3d), but not shorter times (Figure 3f-g, Supplemental Movies 34). Dendrite volume was stable throughout these manipulations, as the critical period was closed (Figure 3e). In sum, astrocyte mitochondria traffic to areas of elevated neuronal activity and away from areas of low neuronal activity to meet local metabolic demand.

\section{Astrocyte-knockdown of tbph/TARDBP enhances mitochondria-synapse association}

The early stages of ALS are characterized by motor neuron hyperexcitability, but not excitotoxicity ${ }^{16}$. During this phase, astrocytes maintain their homeostatic functions and are thought to be neuroprotective ${ }^{14}$. To test whether loss of activity-dependent astrocyte mitochondrial trafficking contributes to motor neuron loss in the tbph ALS model, we visualized astrocyte mitochondria (R25H07-gal4, UAS-mito::GFP) relative to motor neuron dendrites (RN2-lexA, lexAop-Chrimson::tdTomato) and synapses (Brp+) following astrocytespecific knockdown of tbph. To achieve efficient knockdown, we used a well-established and previously published RNA interference line $\left(U A S-t b p h^{\mathrm{RNAi}}\right)^{28-30}$. Astrocyte-specific knockdown of $t b p h$ resulted in a significant increase in mitochondria-synapse association and increased volume of astrocyte mitochondrial volume within the motor neuropil; optogenetic activation further enhanced these phenotypes (Figure 4a-d", quantified in e,f). Importantly, neither genotype nor manipulation impacted overall dendrite volume (Figure 4g). Together, 
these data demonstrate that loss of astrocyte Tbph does not impair mitochondrial recruitment, but instead enhances mitochondrial trafficking to the dendritic domain.

\section{Astrocyte-knockdown of $t b p h / T D P-43$ causes ectopic motor neuron activity}

Thus far, we have shown that motor neuron activation can recruit astrocyte mitochondria to motor synapses (Figure 2), and this phenotype is enhanced following astrocyte knockdown of tbph (Figure 4). Motor neuron hyperexcitability is common in ALS models, including mouse and human-patient derived TARDBP mutant motor neurons ${ }^{31,32}$. Given that astrocyte knockdown of tbph resulted in accumulation of astrocyte mitochondria near motor synapses even without optogenetic stimulation of motor neurons (Figure 4e), we tested whether tbph knockdown in astrocytes alone is sufficient to cause motor neuron hyperexcitability. To test this hypothesis, we assessed calcium signaling within motor neuron dendrites (RN2-lexA, lexAop-GCaMP7s; Figure 5a) in astrocyte tbph knockdown animals (R25H07-gal4, UAS$\left.t b p h^{\mathrm{RNAi}}\right)$. During a 15-minute imaging period, control motor dendrites exhibited a baseline level of spontaneous calcium activity (Figure 5b-d, Supplemental Movie 5). In contrast, knock down of tbph in astrocytes led to a significant increase in the frequency of spontaneous calcium events, as well as an increase in the average amplitude of each event (Figure 5b-e, Supplemental Movie 6).

These data indicate that astrocyte knockdown of tbph is sufficient to cause motor neuron hyperexcitability. In murine ALS models, hyperexcitability is caused by a reduction in the astrocyte excitatory amino acid transporter EAAT2 $2^{33,34}$, which clears $90 \%$ of peri-synaptic glutamate in the adult brain ${ }^{35}$. Note that synapse-associated Drosophila astrocytes primarily express EAAT1, a related glutamate receptor that is the major EAAT subtype expressed 
during mammalian CNS development ${ }^{35}$ and is the only high-affinity glutamate transporter in Drosophila $^{36}$. Previous studies in Drosophila found that eaat1 expression levels are dysregulated in $t b p h$ mutants $^{37}$ and glia-specific overexpression of eaat 1 is sufficient to rescue locomotor defects in $t b p h$ mutant larvae ${ }^{38}$. Similarly, we found a significant reduction in EAAT1 levels on astrocytes following tbph knockdown (Figure 5f-h). These data support the model that loss of $t b p h$ from astrocytes results in a reduction in EAAT1, accumulation of excitatory neurotransmitters within the neuropil, and ultimately hyperexcitability.

\section{Identification of cellular machinery for astrocyte mitochondrial trafficking}

If astrocyte mitochondria are trafficked to motor synapses to meet energetic demand, perhaps enhanced astrocyte mitochondrial trafficking in tbph knockdown conditions prevents hyperexcitability (Figure 5) from turning into excitotoxicity. To test this hypothesis, we first needed to determine how mitochondria are trafficked within astrocytes, which is vastly understudied relative to neurons. We performed astrocyte-specific RNAi knockdown of molecular motor and mitochondrial adaptor proteins using previously published RNAi lines ${ }^{30}$, and visualized astrocyte mitochondria (R25H07-gal4, UAS-mito::GFP, UAS-RNAi) by in vivo time-lapse imaging and in fixed preparations (Supplemental Movies 7-11, Figure 6). We screened eight lines targeting the adaptor protein milton ( $\mathrm{N}=3$ lines), the mitochondrial membrane GTPase miro ( $\mathrm{N}=1$ line, previously linked to astrocyte mitochondrial trafficking ${ }^{39}$ ), as well as the molecular motors kinesin heavy chain ( $\mathrm{N}=2$ lines) and dynein heavy chain $(\mathrm{N}=2$ lines). We found that astrocyte-specific knockdown of milton completely blocked mitochondrial trafficking into the neuropil, without any observable defects in the peri-synaptic localization of astrocyte processes (Supplemental Movie 8, Figure 6a-b", 
quantified in $\mathrm{f}-\mathrm{g}$ ). We also observed significant reductions in mitochondrial trafficking following miro or khc knockdown (Supplemental Movies 9-10, Figure 6c-d", quantified in fg). In neurons, Milton is a mitochondrial adaptor protein that serves as a linker between mitochondria-bound Miro and microtubule-associated Kinesin heavy chain ${ }^{40,41}$. Together, this complex facilitates long-distance, $(+)$ end directed axonal trafficking of mitochondria along microtubules. Conversely, astrocyte knockdown of the (-) end directed microtubule motor protein Dynein heavy chain did not have any impact on mitochondrial trafficking into the neuropil (Supplemental Movie 11, Figure 6e-h). At this developmental stage, astrocyte membranes are rapidly growing into the neuropil to tile in between neuronal synapses ${ }^{20,42}$. Our data suggest that during this developmental stage of rapid astrocyte ingrowth, microtubules are oriented $(+)$ end out to facilitate mitochondrial trafficking into distal perisynaptic processes to meet the metabolic demands of neurons.

\section{Astrocyte mitochondrial trafficking deficits modify locomotor behavior}

Having defined the molecular machinery required for astrocyte mitochondrial trafficking, we next wanted to determine whether increased mitochondria-synapse proximity following astrocyte tbph knockdown is neuroprotective. To that end, we used a high-throughput behavioral rig $^{43}$ to assay a series of locomotor behaviors (Figure 7a) in freely crawling $t b p h^{\mathrm{RNAi}}$, milton $^{\mathrm{RNAi}}$, and double knockdown animals at $72 \mathrm{~h}$ ALH (R25H07-gal4, UAS$R N A i)$. We found that single knockdown of tbph in astrocytes did not have a significant impact on locomotor behavior (Figure 7b-c, quantified in $\mathrm{f}-\mathrm{j}$ ). These data suggest that despite elevated motor neuron activity at $8 \mathrm{~h}$ ALH (Figure 5), homeostatic mechanisms stabilize overall motor circuit activity when astrocyte mitochondria can properly translocate to the 
synapse (Figure 4). Astrocyte knockdown of milton alone caused significant reductions in larval distance traveled due to a combined decrease in crawling speed and duration. We also observed increased bending in milton knockdown animals, a measure of uncoordinated movement (Figure 7d, quantified in f-j). In contrast, when we simultaneously knocked down tbph and milton, we saw a significant increase in crawling speed, duration, and overall traversed distance. In double knockdowns, these phenotypes were still accompanied by increased bending, which occurs in milton, but not tbph, single knockdowns. These data suggest that milton knockdown is the primary driver of the uncoordinated phenotype (Figure 7e, quantified in f-j). Thus, in tbph single knockdown animals where mitochondria-synapse association is preserved, homeostatic mechanisms rebalance overall network activity to prevent developmental hyperexcitability (Figure 5) from manifesting in mature locomotor phenotypes (Figure $7 \mathrm{~b}-\mathrm{c}$, quantified in $\mathrm{f}-\mathrm{j}$ ). When we blocked mitochondrial trafficking via milton $^{\mathrm{RNAi}}$ in $t b p h$ knockdown animals, homeostatic mechanisms were not activated.

Accordingly, ectopic motor signaling in the CNS (Figure 5) corresponded with hyperactive crawling (Figure 7).

\section{Astrocyte mitochondrial trafficking deficits impair synapse maintenance}

Blocking mitochondrial trafficking following tbph knockdown resulted in hyperactivity; we wanted to know if this was sufficient to shift hyperexcitability to excitotoxicity. To that end, we genetically labeled all motor neurons (vglut-lexA, lexAop-myr::GFP) while knocking down tbph, milton, or both in astrocytes (R25H07-gal4, UAS-RNAi). We then performed larval fillet preps to visualize the structure of the neuromuscular junction at $96 \mathrm{~h} \mathrm{ALH}$. We focused our analyses on the dorsal-most muscles where our model motor neurons 
(aCC>muscle DA1; RP2>muscle DA2) and others (U1>muscle DO1; U2>muscle DO2) elaborate their synaptic boutons ${ }^{44}$. Similar to our behavioral analyses, we observed no significant difference in bouton number between control and tbph knockdown animals (Figure 8a-b, e-f), suggesting that homeostatic mechanisms that stabilize the motor circuit (Figure 7bc, quantified in f-j) may act at the level of presynaptic vesicle release ${ }^{45}$. Knockdown of milton alone resulted in a significant increase in synaptic bouton number (Figure $8 \mathrm{c}$, quantified in $\mathrm{f}$ ). Because knockdown of milton resulted in decreased crawling speed and distance (Figure 7d, quantified in $\mathrm{f}-\mathrm{j}$ ), these data suggest that astrocyte knockdown of milton results in reduced motor neuron signaling and a compensatory increase in bouton number, but does not achieve homeostasis. Finally, we observed a significant decrease in total bouton number when we blocked mitochondrial trafficking in tbph knockdown animals (R25H07-gal4, UAS-tbph ${ }^{\mathrm{RNAi}}$, UAS-milton ${ }^{\mathrm{RNAi}}$, Figure 8d, quantified in f). Thus, mitochondria-synapse association is necessary for synapse maintenance following astrocyte knockdown of $t b p h$. In sum, our data support the hypothesis that peri-synaptic localization of astrocyte mitochondria stabilizes motor circuit structure and function in a Drosophila model of ALS.

\section{DISCUSSION}

\section{Astrocyte neurotransmitter turnover and ALS}

In pathological conditions (disease or injury), inflammation drives the transformation of healthy astrocytes into reactive astrocytes (astrogliosis), which are characterized by morphological, transcriptional, and functional changes ${ }^{46}$. Reactive astrocytes were first described over a century ago and are considered a hallmark of neurodegenerative disease ${ }^{47}$. Accordingly, most research on the role of astrocytes in neurodegeneration has focused on the putative toxic effects of reactive astrocytes on compromised neuronal populations (e.g. motor neurons in ALS). One of the best- 
studied ALS models featuring astrogliosis is the G93A variant of superoxide dismutase 1 $\left(S O D 1^{G 93 A}\right)$. SOD1 is an enzyme that reduces cellular levels of the free radical superoxide ${ }^{48}$. Mutations in SOD1 are associated with oxidative stress, mitochondrial dysfunction, inflammation, and motor neuron excitotoxicity due to misregulation of peri-synaptic neurotransmitter concentrations ${ }^{49}$. In murine models, astrocyte-specific overexpression of $S O D 1^{G 93 A}$ causes astrocyte activation, triggers release of proinflammatory cytokines, and is sufficient to induce motor neuron death in vitro and in vivo ${ }^{11,50,51}$. Compound mutations $\left(I L-1 \alpha^{-/-}\right.$ $\mathrm{TNF}^{-/-} \mathrm{Clqa}^{-/-}$) that prevent astrocyte reactivity can significantly slow disease progression (but not onset) and extend the life of $S O D 1^{G 93 A}$ animals ${ }^{17}$. In contrast, transplantation of wild-type astrocytes into the spinal cord of $S O D 1^{G 93 A}$ rats can delay both disease onset and progression ${ }^{18}$. These data implicate astrocyte-induced inflammation as a key determinant of ALS progression, but also point to early neuroprotective roles of wild-type astrocytes.

Here, we wanted to determine which astrocyte functions support motor circuit activity and maintenance in a Drosophila model of ALS. We focused on tbph/TARDBP as a common pathological indicator in ALS/FTD ${ }^{6}$. In mouse, depletion of astrocyte Tardbp is associated with astrocyte reactivity in mature motor circuits where symptoms have already manifested ${ }^{31}$. Although Drosophila astrocytes are not known to become reactive, glia-specific knockdown of tbph is linked to reduced adult lifespan and decreased motor coordination ${ }^{37}$ and glia can modify the severity of ALS phenotypes in $t b p h$ null flies ${ }^{52}$. We focused our initial experiments on presymptomatic stages to determine what homeostatic function(s) of astrocytes are lost following tbph knockdown, and how that in turn impacts motor circuit maintenance. We found that loss of tbph in astrocytes alone enhances motor neuron activity, likely due to reductions in EAAT1 expression on astrocyte membranes and accumulation of extracellular glutamate. The Drosophila 
neuromuscular junction (NMJ) exhibits striking homeostatic plasticity to stabilize motor output in experimental conditions where motor neuron activity is perturbed ${ }^{45,53}$. In accordance with these previous findings, we did not observe overt behavioral defects in astrocyte tbph knockdown animals. In addition, we did not observe any significant changes in synaptic bouton number following astrocyte tbph knockdown. Similarly, depletion of astrocyte Tardbp in mouse does not impact NMJ morphology ${ }^{31}$. These data support the hypothesis that when astrocyte mitochondriasynapse association is intact, homeostatic plasticity mechanisms at the NMJ (e.g. presynaptic release) can compensate for ectopic motor neuron activation in the CNS to stabilize motor output and in turn, locomotor behavior.

\section{Astrocyte-neuron metabolic coupling ensures motor circuit homeostasis}

A primary function of healthy astrocytes is to meet the metabolic demand of associated neurons ${ }^{13,21}$. We found that astrocyte mitochondria sense and respond to local changes in neuronal activity, as stimulation of motor neurons resulted in a rapid mobilization and then stabilization of astrocyte mitochondria near motor synapses. These findings are consistent with previous characterizations of activity-dependent mitochondrial trafficking in hippocampal slice cultures $^{27}$. We questioned whether knockdown of tbph would impair activity-dependent mitochondrial trafficking. Instead, we observed enhanced localization of peri-synaptic astrocyte mitochondria following knockdown of $t b p h$, theoretically to meet the energetic demand of hyperactive motor neurons. Indeed, when we blocked mitochondrial trafficking in tbph knockdown animals, we observed significant increases in crawling speeds that preceded synapse loss at the NMJ. Thus, in the absence of peri-synaptic astrocyte mitochondria, homeostatic mechanisms that stabilize motor circuit activity failed to engage, resulting in excitotoxicity. 


\section{Do astrocytes dampen neuronal activity?}

Hyperexcitability is a major cause of motor neuron death in $\operatorname{ALS}^{16}$. We found that knockdown of astrocyte $t b p h$ causes enhanced motor neuron activity due to decreased astrocyte EAAT1 expression. Similarly, overexpression of SOD $1{ }^{\mathrm{G} 93 \mathrm{~A}}$ in astrocytes results in motor neuron hyperexcitability due to decreased expression of EAAT2 ${ }^{54-56}$. These data indicate that wild-type astrocytes generally serve to restrict motor neuron activity to a given physiological range. Interestingly, we recently identified a critical period of activity-dependent homeostatic plasticity in the Drosophila motor circuit that is closed by astrocyte-motor neuron contact. Within this window, motor neurons scale dendrite length, complexity, and connectivity in response to their own activity; failure to close the critical period results in sustained changes to locomotor behavior $^{20}$. In the present study, we found that in a Drosophila model of ALS, blocking astrocyte mitochondrial transport impaired homeostatic mechanisms that rebalance motor circuit activity during development, resulting in mature synapse loss. These findings open up the intriguing possibility that astrocytes buffer developmental activity to facilitate long-term circuit maintenance and function, and that ultimately, neurodegenerative diseases reflect failed checks on developmental activity. 


\section{Acknowledgements}

We thank Marc Freeman for providing fly stocks. We thank members of the Doe lab for comments on the manuscript. Stocks obtained from the Bloomington Drosophila Stock Center were used in this study. Funding was provided by HHMI (CQD), R01 HD27056 (CQD), NIH K99NS121137 (SDA). SDA is a Milton Safenowitz Post-doctoral fellow of the ALSA.

\section{Author Contributions}

SDA conceived of the project; SDA performed and analyzed all experiments in Figures 1-4 and 6; SDA and SAZ performed and analyzed experiments in Figures 5-8; ELH analyzed GCaMP7s data in Figure 5; CQD provided feedback during the project; SDA, SAZ, and ELH wrote the paper and prepared the figures. All authors commented and approved of the manuscript.

\section{Competing Interest Statement}

The authors declare no competing financial or non-financial interests.

\section{Data Availability Statement}

This study did not generate/analyze datasets/code. Raw data for any main or supplemental figure (.lsm, .czi, or .avi files) can be supplied upon request. 


\section{REFERENCES}

1. Amyotrophic Lateral Sclerosis. (Exon Publications, 2021).

doi:10.36255/exonpublications.amyotrophiclateralsclerosis.2021.

2. Rosen, D. R. et al. Mutations in $\mathrm{Cu} / \mathrm{Zn}$ superoxide dismutase gene are associated with familial amyotrophic lateral sclerosis. Nature 362, 59-62 (1993).

3. Rowland, L. P. \& Shneider, N. A. Amyotrophic Lateral Sclerosis. N. Engl. J. Med. 344, 1688-1700 (2001).

4. Lattante, S., Rouleau, G. A. \& Kabashi, E. TARDBP and FUS Mutations Associated with Amyotrophic Lateral Sclerosis: Summary and Update. Hum. Mutat. 34, 812-826 (2013).

5. Mejzini, R. et al. ALS Genetics, Mechanisms, and Therapeutics: Where Are We Now? Front. Neurosci. 13, 1310 (2019).

6. Neumann, M. et al. Ubiquitinated TDP-43 in frontotemporal lobar degeneration and amyotrophic lateral sclerosis. Science 314, 130-133 (2006).

7. Janssens, J. \& Van Broeckhoven, C. Pathological mechanisms underlying TDP-43 driven neurodegeneration in FTLD-ALS spectrum disorders. Hum. Mol. Genet. 22, R77-R87 (2013).

8. Wegorzewska, I. \& Baloh, R. H. TDP-43-based animal models of neurodegeneration: new insights into ALS pathology and pathophysiology. Neurodegener. Dis. 8, 262-274 (2011).

9. Vahsen, B. F. et al. Non-neuronal cells in amyotrophic lateral sclerosis - from pathogenesis to biomarkers. Nat. Rev. Neurol. 17, 333-348 (2021).

10. Lee, Y. et al. Oligodendroglia metabolically support axons and contribute to neurodegeneration. Nature 487, 443-448 (2012). 
11. Papadeas, S. T., Kraig, S. E., O’Banion, C., Lepore, A. C. \& Maragakis, N. J. Astrocytes carrying the superoxide dismutase 1 (SOD1G93A) mutation induce wild-type motor neuron degeneration in vivo. Proc. Natl. Acad. Sci. U. S. A. 108, 17803-17808 (2011).

12. Joshi, A. U. et al. Fragmented mitochondria released from microglia trigger A1 astrocytic response and propagate inflammatory neurodegeneration. Nat. Neurosci. 22, 1635-1648 (2019).

13. Lago-Baldaia, I., Fernandes, V. M. \& Ackerman, S. D. More Than Mortar: Glia as Architects of Nervous System Development and Disease. Front. Cell Dev. Biol. 8, 611269 (2020).

14. Izrael, M., Slutsky, S. G. \& Revel, M. Rising Stars: Astrocytes as a Therapeutic Target for ALS Disease. Front. Neurosci. 14, 824 (2020).

15. Lee, J. et al. Astrocytes and Microglia as Non-cell Autonomous Players in the Pathogenesis of ALS. Exp. Neurobiol. 25, 233-240 (2016).

16. Do-Ha, D., Buskila, Y. \& Ooi, L. Impairments in Motor Neurons, Interneurons and Astrocytes Contribute to Hyperexcitability in ALS: Underlying Mechanisms and Paths to Therapy. Mol. Neurobiol. 55, 1410-1418 (2018).

17. Guttenplan, K. A. et al. Knockout of reactive astrocyte activating factors slows disease progression in an ALS mouse model. Nat. Commun. 11, 3753 (2020).

18. Lepore, A. C. et al. Focal transplantation-based astrocyte replacement is neuroprotective in a model of motor neuron disease. Nat. Neurosci. 11, 1294-1301 (2008).

19. Casci, I. \& Pandey, U. B. A fruitful endeavor: modeling ALS in the fruit fly. Brain Res. 1607, 47-74 (2015).

20. Ackerman, S. D., Perez-Catalan, N. A., Freeman, M. R. \& Doe, C. Q. Astrocytes close a motor circuit critical period. Nature 592, 414-420 (2021). 
21. Tsacopoulos, M. \& Magistretti, P. J. Metabolic coupling between glia and neurons. J. Neurosci. Off. J. Soc. Neurosci. 16, 877-885 (1996).

22. Suzuki, A. et al. Astrocyte-neuron lactate transport is required for long-term memory formation. Cell 144, 810-823 (2011).

23. Benjamin Kacerovsky, J. \& Murai, K. K. Stargazing: Monitoring subcellular dynamics of brain astrocytes. Neuroscience 323, 84-95 (2016).

24. Landgraf, M., Jeffrey, V., Fujioka, M., Jaynes, J. B. \& Bate, M. Embryonic origins of a motor system: motor dendrites form a myotopic map in Drosophila. PLoS Biol. 1, E41 (2003).

25. Sales, E. C., Heckman, E. L., Warren, T. L. \& Doe, C. Q. Regulation of subcellular dendritic synapse specificity by axon guidance cues. eLife 8, e43478 (2019).

26. Jackson, J. G. \& Robinson, M. B. Reciprocal Regulation of Mitochondrial Dynamics and Calcium Signaling in Astrocyte Processes. J. Neurosci. Off. J. Soc. Neurosci. 35, 15199$15213(2015)$.

27. Jackson, J. G., O’Donnell, J. C., Takano, H., Coulter, D. A. \& Robinson, M. B. Neuronal activity and glutamate uptake decrease mitochondrial mobility in astrocytes and position mitochondria near glutamate transporters. J. Neurosci. Off. J. Soc. Neurosci. 34, 1613-1624 (2014).

28. Maccioni, R. et al. Standardized phytotherapic extracts rescue anomalous locomotion and electrophysiological responses of TDP-43 Drosophila melanogaster model of ALS. Sci. Rep. 8, 16002 (2018).

29. Kushimura, Y. et al. Overexpression of ter94, Drosophila VCP, improves motor neuron degeneration induced by knockdown of TBPH, Drosophila TDP-43. Am. J. Neurodegener. Dis. 7, 11-31 (2018). 
30. Perkins, L. A. et al. The Transgenic RNAi Project at Harvard Medical School: Resources and Validation. Genetics 201, 843-852 (2015).

31. Yang, C. et al. Partial loss of TDP-43 function causes phenotypes of amyotrophic lateral sclerosis. Proc. Natl. Acad. Sci. U. S. A. 111, E1121-1129 (2014).

32. Devlin, A.-C. et al. Human iPSC-derived motoneurons harbouring TARDBP or C9ORF72 ALS mutations are dysfunctional despite maintaining viability. Nat. Commun. 6, 5999 (2015).

33. Sasaki, S., Komori, T. \& Iwata, M. Excitatory amino acid transporter 1 and 2 immunoreactivity in the spinal cord in amyotrophic lateral sclerosis. Acta Neuropathol. (Berl.) 100, 138-144 (2000).

34. Lapucci, A. et al. Effect of Class II HDAC inhibition on glutamate transporter expression and survival in SOD1-ALS mice. Neurosci. Lett. 656, 120-125 (2017).

35. Kim, K. et al. Role of excitatory amino acid transporter-2 (EAAT2) and glutamate in neurodegeneration: opportunities for developing novel therapeutics. J. Cell. Physiol. 226, 2484-2493 (2011).

36. Besson, M. T., Soustelle, L. \& Birman, S. Identification and structural characterization of two genes encoding glutamate transporter homologues differently expressed in the nervous system of Drosophila melanogaster. FEBS Lett. 443, 97-104 (1999).

37. Diaper, D. C. et al. Drosophila TDP-43 dysfunction in glia and muscle cells cause cytological and behavioural phenotypes that characterize ALS and FTLD. Hum. Mol. Genet. 22, 38833893 (2013).

38. Romano, G. et al. Glial TDP-43 regulates axon wrapping, GluRIIA clustering and fly motility by autonomous and non-autonomous mechanisms. Hum. Mol. Genet. 24, 6134-6145 (2015). 
39. Stephen, T.-L. et al. Miro1 Regulates Activity-Driven Positioning of Mitochondria within Astrocytic Processes Apposed to Synapses to Regulate Intracellular Calcium Signaling. J. Neurosci. Off. J. Soc. Neurosci. 35, 15996-16011 (2015).

40. Glater, E. E., Megeath, L. J., Stowers, R. S. \& Schwarz, T. L. Axonal transport of mitochondria requires milton to recruit kinesin heavy chain and is light chain independent. $J$. Cell Biol. 173, 545-557 (2006).

41. Schwarz, T. L. Mitochondrial trafficking in neurons. Cold Spring Harb. Perspect. Biol. 5, a011304 (2013).

42. Stork, T., Sheehan, A., Tasdemir-Yilmaz, O. E. \& Freeman, M. R. Neuron-glia interactions through the Heartless FGF receptor signaling pathway mediate morphogenesis of Drosophila astrocytes. Neuron $\mathbf{8 3}, 388-403$ (2014).

43. Risse, B. et al. FIM, a novel FTIR-based imaging method for high throughput locomotion analysis. PloS One 8, e53963 (2013).

44. Landgraf, M., Bossing, T., Technau, G. M. \& Bate, M. The origin, location, and projections of the embryonic abdominal motorneurons of Drosophila. J. Neurosci. Off. J. Soc. Neurosci. 17, 9642-9655 (1997).

45. Goel, P. \& Dickman, D. Synaptic homeostats: latent plasticity revealed at the Drosophila neuromuscular junction. Cell. Mol. Life Sci. CMLS 78, 3159-3179 (2021).

46. Escartin, C. et al. Reactive astrocyte nomenclature, definitions, and future directions. Nat. Neurosci. 24, 312-325 (2021).

47. Andriezen, W. L. The Neuroglia Elements in the Human Brain. Br. Med. J. 2, 227-230 (1893).

48. Fridovich, I. Superoxide dismutases. Adv. Enzymol. Relat. Areas Mol. Biol. 58, 61-97 (1986). 
49. Barber, S. C., Mead, R. J. \& Shaw, P. J. Oxidative stress in ALS: a mechanism of neurodegeneration and a therapeutic target. Biochim. Biophys. Acta 1762, 1051-1067 (2006).

50. Cassina, P. et al. Mitochondrial dysfunction in SOD1G93A-bearing astrocytes promotes motor neuron degeneration: prevention by mitochondrial-targeted antioxidants. J. Neurosci. Off. J. Soc. Neurosci. 28, 4115-4122 (2008).

51. Nagai, M. et al. Astrocytes expressing ALS-linked mutated SOD1 release factors selectively toxic to motor neurons. Nat. Neurosci. 10, 615-622 (2007).

52. Romano, G. et al. Downregulation of glutamic acid decarboxylase in Drosophila TDP-43-null brains provokes paralysis by affecting the organization of the neuromuscular synapses. Sci. Rep. 8, 1809 (2018).

53. Paradis, S., Sweeney, S. T. \& Davis, G. W. Homeostatic control of presynaptic release is triggered by postsynaptic membrane depolarization. Neuron 30, 737-749 (2001).

54. Howland, D. S. et al. Focal loss of the glutamate transporter EAAT2 in a transgenic rat model of SOD1 mutant-mediated amyotrophic lateral sclerosis (ALS). Proc. Natl. Acad. Sci. U. S. A. 99, 1604-1609 (2002).

55. Guo, H. et al. Increased expression of the glial glutamate transporter EAAT2 modulates excitotoxicity and delays the onset but not the outcome of ALS in mice. Hum. Mol. Genet. 12, 2519-2532 (2003).

56. Rothstein, J. D. et al. Beta-lactam antibiotics offer neuroprotection by increasing glutamate transporter expression. Nature 433, 73-77 (2005). 


\section{METHODS}

\section{Lead Contact and Materials Availability}

Additional information and inquiries regarding resources and reagents should be directed to and will be fulfilled upon request by Lead Contact Sarah D Ackerman (sarah.ackerman@wustl.edu).

\section{Experimental Model and Subject Details}

\section{Fly husbandry}

We raised all fly stocks at $25^{\circ} \mathrm{C}$ on standard cornmeal fly food. Animals were staged relative to a $25^{\circ} \mathrm{C}$ standard. At $25^{\circ} \mathrm{C}$, embryos take 21 hours to hatch into larvae ${ }^{57}$; larvae hatch and develop $1.25 \mathrm{x}$ faster at $30^{\circ} \mathrm{C}$, and $2 \mathrm{x}$ slower at $18^{\circ} \mathrm{C}$.

Transgenes (in order of appearance)

1. $\quad R N 2-l e x A^{20}$

2. 13lexAop-CsChrimson::tdTomato ${ }^{20}$

3. R25H07-gal4 (BDSC\# 49145)

4. UAS-mito-HA-GFP/cyo (BDSC\# 8442)

5. 13xLexAop2-CD4-tdTom/T6b (BDSC\# 77139)

6. RN2-gal4 ${ }^{24}$

7. UAS-GtACR2::EYFP ${ }^{58}$

8. $\quad$ alrm-lexA::GAD (Chromosome 3$)^{59}$

9. lexAop-mCherry::mito.OMM (BDSC\# 66530)

10. 10XUAS-myr::GFP (BDSC\# 32198) 
506

11. UAS-tbph $h^{\mathrm{RNAi}}(\mathrm{BDSC} \# 39014)$

12. UAS-mito-HA-GFP (chromosome 3, BDSC\# 8443)

13. UAS-luciferase ${ }^{\mathrm{RNAi}}$ (BDSC\# 31603)

14. 13XLexAop-IVS-jGCaMP7s (BDSC\# 80913)

15. alrm-gal4 (Chromosome 2) $)^{59}$

16. 10XUAS-mcd8::GFP (BDSC\# 5137)

17. UAS-milton ${ }^{\mathrm{RNAi}}$ (BDSC\#s 28385, 43173, 44477)

18. $U A S-k h c^{\mathrm{RNAi}}(\mathrm{BDSC} \# \mathrm{~s} 35409,35770)$

19. $\quad U A S-$ miro $^{\mathrm{RNAi}}$ (BDSC\# 43973)

20. UAS-Dhc64C (BDSC\# 36583, 36698)

21. Mi\{Trojan-lexA:QFAD.2\}VGlut[MI04979-TlexA:QFAD.2]/CyO (BDSC\# 60314)

22. 13XLexAop2-IVS-myr::GFP (X Chromosome, BDSC\# 32211)

\section{$\underline{\text { Animal collections }}$}

For imaging of wild-type brains without optogenetics (Figures 2-3): We reared crosses at $25^{\circ} \mathrm{C}$ in collection bottles fitted with $3.0 \%$ agar apple juice caps seeded with plain yeast paste (Baker's year + water). We then collected embryos on fresh 3.0\% agar apple juice caps seeded with plain yeast paste for 1.5 hours (h) and aged at $25^{\circ} \mathrm{C}$ until hatching. At $0 \mathrm{~h}$ after larval hatching (ALH), larvae were collected and transferred to fresh apple caps (N=20 larvae per cap) containing yeast paste and aged until $8 \mathrm{~h} \mathrm{ALH,} \mathrm{when} \mathrm{they} \mathrm{were} \mathrm{dissected} \mathrm{and}$ prepared for imaging. 
For imaging of RNAi brains without optogenetics (Figures 4-6, 8): We reared crosses at $25^{\circ} \mathrm{C}$ in collection bottles fitted with 3.0\% agar apple juice caps seeded with plain yeast paste (Baker's year + water). We then collected embryos on fresh 3.0\% agar apple juice caps seeded with plain yeast paste for 1.5 hours (h) and aged at $30^{\circ} \mathrm{C}$ until hatching to maximize RNAi expression. For assessment of mitochondrial trafficking, larvae were collected at $0 \mathrm{~h}$ ALH and transferred to apple caps ( $\mathrm{N}=20$ larvae per cap) containing fresh year paste, aged 6.5 $\mathrm{h}\left(\sim 8 \mathrm{~h} \mathrm{ALH}\right.$ at $25^{\circ} \mathrm{C}$ standard $)$, when they were dissected and prepared for imaging. For assessment of synapse number at the neuromuscular junction, apple caps were transferred to fresh food bottles (corn meal) when animals reached $0 \mathrm{~h}$ ALH. Bottles were maintained at $30^{\circ} \mathrm{C}$ for $77 \mathrm{~h}\left(\sim 96 \mathrm{~h} \mathrm{ALH}\right.$ at $25^{\circ} \mathrm{C}$ standard $)$, when larvae were collected, dissected, and prepared for imaging.

For optogenetics on control animals (Figures 2-3): We reared crosses at $25^{\circ} \mathrm{C}$ in collection bottles fitted with 3.0\% agar apple juice caps seeded with fresh yeast paste that was supplemented with 0.5mM all-trans retinal (+ATR) (Sigma-Aldrich, R2500-100MG). Crosses were supplied fresh yeast paste daily (+ATR) for a minimum of $72 \mathrm{~h}$ prior to embryo collection to ensure maternal transfer of ATR into embryos. We then collected embryos on $3.0 \%$ agar apple juice caps with yeast paste (+ATR) for $1.5 \mathrm{~h}$ and aged at $25^{\circ} \mathrm{C}$. To prevent premature optogenetic activation, we kept all crosses, embryos, and larvae in dark conditions until the appropriate developmental stage. At $25^{\circ} \mathrm{C}$, Drosophila embryos hatch at $21 \mathrm{~h}$ after egg laying (AEL). Larvae were collected at $0 \mathrm{~h} \mathrm{ALH}$ and transferred to fresh apple caps $(\mathrm{N}=10$ larvae per cap) containing yeast paste (+ATR). For fixed preparations, larvae were then transferred to activating/silencing conditions for 1 hour and dissected at $8 \mathrm{~h} \mathrm{ALH.} \mathrm{For}$ 
live imaging experiments, larvae were dissected at $8 \mathrm{~h} \mathrm{ALH}$ and transferred to the confocal microscope for manipulation/recording (read more below).

For optogenetics with RNAi (Figure 4): We reared crosses at $25^{\circ} \mathrm{C}$ in collection bottles fitted with 3.0\% agar apple juice caps seeded with fresh yeast paste that was supplemented with 0.5mM all-trans retinal (+ATR) (Sigma-Aldrich, R2500-100MG). Crosses were supplied fresh yeast paste daily (+ATR) for a minimum of $72 \mathrm{~h}$ prior to embryo collection to ensure maternal transfer of ATR into embryos. We then collected embryos on 3.0\% agar apple juice caps with yeast paste (+ATR) for $1.5 \mathrm{~h}$ and aged at $30^{\circ} \mathrm{C}$. To prevent premature optogenetic activation, we kept all crosses, embryos, and larvae in dark conditions until the appropriate developmental stage. At $0 \mathrm{~h}$ ALH, we transferred larvae to fresh apple caps $(\mathrm{N}=10$ larvae per cap) containing yeast paste (+ATR) and aged $6.5 \mathrm{~h}\left(\sim 8 \mathrm{~h}\right.$ ALH at $25^{\circ} \mathrm{C}$ standard) prior to dissection for dark-reared controls, or aged $5.5 \mathrm{~h}$ followed by $1 \mathrm{~h}$ light activation. All animals were dissected immediately following activity manipulation.

For behavioral analyses: We reared crosses at $25^{\circ} \mathrm{C}$ in collection bottles fitted with $3.0 \%$ agar apple juice caps containing plain yeast paste. Embryos were then collected on $3.0 \%$ agar apple juice caps with plain yeast paste for $1.5 \mathrm{~h}$ and aged at $30^{\circ} \mathrm{C}$. Apple caps were transferred to fresh food bottles (corn meal) when animals reached $0 \mathrm{~h} \mathrm{ALH}$. Bottles were maintained at $30^{\circ} \mathrm{C}$ for $60 \mathrm{~h}\left(\sim 72 \mathrm{~h}\right.$ ALH at $25^{\circ} \mathrm{C}$ standard $)$ until larvae reached wandering $3^{\text {rd }}$ instar stage, when they were transferred to the behavioral rig for testing. 


\section{METHOD DETAILS}

\section{Sustained optogenetic activation/silencing}

We used the following published strategy ${ }^{20}$ for optogenetic activation/silencing in all fixed preparation experiments (Figures 2-4). At the designated stage, dark-reared larvae (maintained on apple caps with yeast + ATR) were placed beneath a full spectrum light bulb, shining with an average intensity of $10550 \mathrm{~lx}$ (determined with two independent photometer software programs developed for Android: Light Meter@ and Photometer@). We then dissected larvae in low-light conditions ( $<100 \mathrm{~lx})$ immediately following activity manipulations. Yeast paste was placed in the middle of the apple cap to motivate larvae to remain on the top of the agar plate throughout the duration of the manipulation. Any larvae found outside of this area were not processed further.

\section{Immunohistochemistry}

Larval brains were dissected in sterile-filtered, ice-cold 1X PBS and mounted on 12mm \#1 thickness poly-D-lysine coated round coverslips (Corning ${ }^{\circledR}$ BioCoat $^{\mathrm{TM}}$, 354085 or German Glass Cover Slips, Fisher Scientific, 72196-12). We fixed brains for 12 minutes in fresh 4\% paraformaldehyde (Electron Microscopy Sciences, 15710) in .3\% PBSTriton, and then washed in .3\% PBSTriton to remove fixative. For larval fillet preps, we dissected larvae in sterile-filtered, ice-cold HL3.1, fixed for 45 minutes in fresh $4 \%$ paraformaldehyde in detergent-free 1X PBS (Electron Microscopy Sciences, 15710), and then washed in .3\% PBSTriton to remove fixative. At this point, all samples were treated the same way through post-secondary washes. Samples were blocked overnight at $4{ }^{\circ} \mathrm{C}$ in $.3 \%$ PBSTriton supplemented with 1\% BSA (Fisher, BP1600-100), 1\% normal donkey serum and 1\% normal 
goat serum (Jackson ImmunoResearch Laboratories, Inc., 017-000-121 and 005-000-121).

Samples were then incubated in primary antibody for one-two days at $4^{\circ} \mathrm{C}$. The primary was removed, and brains were washed overnight at $4^{\circ} \mathrm{C}$ with $0.3 \%$ PBSTriton. Samples were then incubated in secondary antibodies overnight at $4{ }^{\circ} \mathrm{C}$. The secondary antibodies were removed, and samples transferred to .3\% PBSTriton overnight. Larval brains were then prepared for DPX mounting. Brains were dehydrated with an ethanol series: $30 \%, 50 \%, 70 \%, 90 \%$, each for 5 minutes, then twice in $100 \%$ ethanol for 10 minutes each (Decon Labs, Inc., 2716GEA). Finally, samples were incubated in xylenes (Fisher Chemical, X5-1) for 2 x 10 minutes, were mounted onto slides containing DPX mountant (Millipore Sigma, 06552), and cured for 1-2 days before imaging. After post-secondary .3\% PBSTriton washes, larval fillet preps were washed into 1X PBS overnight. Fillet preps were then incubated in Phalloidin-405 (Alexa Fluor $^{\text {TM }}$ Plus 405 Phalloidin, a30104) in 1X PBS for 5 minutes, washed back into 1X PBS, and mounted in ProLong Glass Antifade Mountant (Thermo Fisher Scientific, P36982).

The following primary and secondary antibodies were used:

$\begin{array}{lll}\text { Primary Antibody } & \text { Source } & \text { Figures }\end{array}$ (concentration)

$\begin{array}{lll}\text { Chicken anti-GFP }(1: 1000) & \text { Aves Cat. GFP-1010 } & 1-5,8\end{array}$

$\begin{array}{lll}\text { Rabbit anti-dsred (1:500) } \quad \text { Takara Bio Cat. } 632496 & 1-2,4\end{array}$

Rabbit anti-cherry (1:500)

Novus Biologicals, Cat. NBP2-

1,3

25157 


\begin{tabular}{lll}
\hline Ms anti-Brp/Nc82 (1:100) DSHB nc82 $\quad 1-4,6$
\end{tabular}

\begin{tabular}{ll}
\hline Rabbit anti-EAAT1 (1:5000) $\quad$ D. Van Meyel lab & 60
\end{tabular}

\begin{tabular}{ll}
\hline Rabbit anti-Gat (1:4000) M. Freeman lab $^{42} \quad$ M
\end{tabular}

\begin{tabular}{lll}
\hline Ms anti-Dlg $(1: 100)$ & DSHB $4 F 3$ & 8
\end{tabular}

\begin{tabular}{lll}
\hline Phalloidin-405 (1:40) & Alexa Fluor & TM, a30104
\end{tabular}

Secondary Antibodies:

All secondary antibodies were purchased from Jackson ImmunoResearch and used at a working concentration of 1:400. The following antibodies were used: Alexa Fluor® Rhodamine RedTM-X Donkey-Anti Mouse (715-295-151), Alexa Fluor® 647 AffiniPure Donkey Anti-Mouse IgG (715-605-151), Alexa Fluor® 488 Donkey anti-Chicken (703-545-

155), Alexa Fluor® 647 Donkey-Anti Rabbit (711-605-152); Alexa Fluor® 488 Donkey anti-

Rabbit (711-545-152), Alexa Fluor® Rhodamine RedTM-X Donkey Anti-Rabbit (711-295152).

\section{Light Microscopy}

Fixed larval preparations for synapse quantifications (central and NMJ, Figures 1-4, 8) and all live imaging experiments (Figures 2-5, Supplemental Movies 1-11) were imaged on a Zeiss LSM 800 laser scanning confocal fitted with a 63x/1.40 NA Oil Plan-Apochromat DIC m27 objective lens and GaAsP photomultiplier tubes. Fixed larval preparations for astrocyte morphology analyses (Figure 6) were imaged with a Zeiss LSM 700 laser scanning confocal using a 63x/1.4 NA Oil Plan-Apochromat DIC m27 objective lens. 
Image processing and analyses

Quantitative analyses were performed either in Imaris 9.2.0 or higher (Bitplane 9.2.0, Figures 1-4, 6) or in FIJI (ImageJ 1.50d, https://imagej.net/Fiji, Figure 5, 8).

\section{$\underline{\text { Time-lapse imaging of fictive preparations }}$}

The following assay was used for live imaging of aCC/RP2 dendrites and/or astrocyte mitochondria in isolated CNS (Figures 2-3, 5, Supplemental Movies 1-11). Larvae were dissected at $8 \mathrm{~h}$ ALH in a hemolymph-like solution (HL3.1); both lobes and the ventral nerve cord were kept intact. Isolated brains were mounted dorsal-side down on a $12 \mathrm{~mm} \# 1$ thickness poly-D-lysine coated round coverslips (Corning ${ }^{\circledR}$ BioCoat ${ }^{\mathrm{TM}}$, 354085 or German Glass Cover Slips, Fisher Scientific, 72196-12). The cover slip was then inverted onto a slide with vacuum grease as a bridge and pressed down until brain lobes were sandwiched between the slide and cover glass. Samples that were damaged during mounted were not imaged. Samples were imaged on a Zeiss LSM 800 laser scanning confocal fitted with a 63x/1.40 NA Oil PlanApochromat DIC m27 objective lens and GaAsP photomultiplier tubes, and imaged using a $488 \mathrm{~nm}$ laser and/or $561 \mathrm{~nm}$ laser when appropriate. For optogenetic experiments (Figures 2$3)$, both dissections and mounting were performed under low light conditions ( $<100 \mathrm{~lx})$ to delay optogenetic activation. Continuous scans were obtained every 30 seconds for 15 minutes, with two hemisegments in the field of view. A z-stack of $12 \mu \mathrm{m}$ (allowing for drift in Z) with $.5 \mu \mathrm{m}$ step size was performed. Finally, we imaged abdominal segments 5-6, which were less prone to drift over the 15-minute acquisition. For GCaMP7s assays (Figure 5), a zstack of $19 \mu \mathrm{m}$ (allowing for drift in Z) with $1 \mu \mathrm{m}$ step size was imaged continuously with stacks acquired every 15 seconds for 15 minutes, with abdominal segments 3-7 in view. For 
652

our mitochondria machinery screen (Supplemental Movies 7-11), we obtained $50 \mu \mathrm{m}$ stacks (step size of $1 \mu \mathrm{m}$ ) once a minute for 15 minutes, with abdominal segments 5-8 in view.

\section{Behavioral analyses}

We transferred larvae at $72 \mathrm{~h} \mathrm{ALH} \mathrm{from} \mathrm{food} \mathrm{bottles} \mathrm{(standard} \mathrm{corn} \mathrm{meal)} \mathrm{to} 1.2 \%$ agarose plates for half an hour prior to locomotion assays to allow the larvae to acclimate to the new crawling surface. Larvae were then transferred to a FIM behavior table ${ }^{43}$ fitted with a fresh $1.2 \%$ agarose gel and allowed to further acclimate for two minutes prior to imaging. Two or more independent cohorts of larvae ( $\mathrm{N}=30$ larvae per cohort) were tested per genotype. Larval crawling was imaged at $4 \mathrm{~Hz}, 91$ pixels/cm for one minute using a Basler acA2040-25gm camera in the Pylon5 Camera Software Suite (Basler). Data were then analyzed using FIMTrack software using standard settings ${ }^{43}$. Data files were blinded to genotype until after analysis.

\section{Figure preparation}

Images in figures were prepared in Imaris (Bitplane AG, version 9.2.0 or higher) or in Fiji (ImageJ 1.50d). Both software packages were used for rendering 3D, max, or orthogonal projections of the data, which were then assembled using Adobe Illustrator. Schematics were drawn in Microsoft Powerpoint.

\section{QUANTIFICATION AND STATISTIC ANALYSIS}

Synapse quantification: association of astrocyte mitochondria with presynapses aCC/RP2-associated presynapses 
For quantification of astrocyte mitochondria in close proximity to aCC/RP2-associated presynapses (Figures 1-4), data were acquired with a voxel size of $.099 \times .099 \times .23 \mu \mathrm{m}^{3}$ and de-convoluted in Imaris. First, aCC/RP2 dendrites within a single hemisegment were reconstructed using the Imaris "Surface" module (no smoothing, thresholds varied with fluorescence intensity). A standard ROI spanned $100 \times 100$ pixels in $\mathrm{XY}$, and $7 \mu \mathrm{m}$ in $\mathrm{Z}$ with the top of the ROI beginning dorsally at the aCC/RP2 axons. The dendrite "Surface" was also used to determine dendrite volume. A distance transformation was then performed on the dendrite "Surface". Second, Brp+ presynapses within the ROI were annotated using the Imaris "Spots" functions and then classified as verified post-synapses if they fell within 90 $\mathrm{nm}$ of the "Surface" (accounts for chromatic aberration and the size of the synaptic cleft) based on previously validated criteria ${ }^{20,25}$, and were used to build a new channel. Third, astrocyte mitochondria within the same ROI were reconstructed using the Imaris "Surface" module (.1 $\mu \mathrm{m}$ smoothing, thresholds varied with fluorescence intensity). A distance transformation was then performed on the astrocyte mitochondria "Surface". Finally, verified presynapse "Spots" were classified as mitochondria-associated if they fell within $120 \mathrm{~nm}$ of the astrocyte mitochondria "Surface" (accounts for chromatic aberration ${ }^{25}$, the thickness of the astrocyte plasma membrane $e^{61}$, and the thickness of both mitochondrial membranes ${ }^{62}$ ).

\section{Astrocyte association with all presynapses}

For quantification of astrocyte membrane and mitochondria-associated presynapses (Figure 6), data were acquired with a voxel size of $.2 \times .2 \times .39 \mu \mathrm{m}^{3}$. Imaris reconstructions were applied to the entire larval brain. First, we reconstructed astrocyte membranes using the Imaris "Surface" module (no smoothing, thresholds varied with fluorescence intensity). A 
distance transformation was performed on the membrane "Surface." Then, we constructed astrocyte mitochondria using the Imaris "Surface" module (no smoothing, thresholds varied with fluorescence intensity). A second distance transformation was performed on the mitochondria "Surface." Finally, Brp+ presynapses were annotated using the Imaris "Spots" functions and then categorized by proximity $(\leq 1 \mu \mathrm{m})$ to the astrocyte mitochondria or membrane "Surface."

\section{Analysis of time-lapse imaging samples (optogenetics)}

For analysis of mitochondrial dynamics over time (Figures 2-3), data were collected with a voxel size of $.085 \times .085 \times .5 \mu \mathrm{m}^{3}$. Any samples that drifted out of the frame of view during the acquisition period were not processed further. Data were then imported to Imaris and analyzed using the "Surface" module $(.1 \mu \mathrm{m}$ smoothing, thresholding varied by fluorescence intensity of sample). A standard ROI spanned 200 x 200 pixels in XY, and $12.5 \mu \mathrm{m}$ in Z. First, we built a "Surface" to mask the motor dendrites. Then, we built a "Surface" to mask the astrocyte mitochondria. We then used the "Surface-Surface" overlap function (Imaris 9.7.0 or later) to calculate the volume of all mitochondria within the dendritic domain $(\leq 120$ $\mathrm{nm}$ from motor dendrites), and to determine the total volume of "Surface" overlap (aka volume of membrane overlap).

Analysis of GCaMP imaging data

Image files were blinded to genotype. Raw time-lapse image stacks were imported into Fiji and converted to time-lapse maximum intensity projections. These converted files were loaded into a custom MATLAB script ${ }^{25}$. The script first performs rigid registration to correct for movement artifacts during recording, and then allows for ROI selection. ROIs were drawn around aCC/RP2 
721

dendrites in 6 individual hemisegments, 3 per side. ROI size was constant across all analyzed files (Figure 5). The script then quantifies and plots the frame-by-frame average dF/F within each ROI. To identify peaks of calcium activity in an unbiased manner, our $\mathrm{dF} / \mathrm{F}$ traces were next loaded into the MATLAB program PeakCaller ${ }^{63}$. In PeakCaller, the following parameters were specified for the program to identify peaks in each trace: Required Rise $=20 \%$, Required Fall $=$ 30\%, Max Lookback and Lookahead pts $=10$ frames. Peaks $<\mathrm{dF} / \mathrm{F}=0.1$ were excluded from analysis. We used PeakCaller to identify the number of calcium peaks in each $\mathrm{dF} / \mathrm{F}$ trace. We logged the amplitude of each peak $(\mathrm{dF} / \mathrm{F})$ within a trace, and averaged these amplitudes together to obtain a single average peak fluorescence value for each ROI.

After quantifying the frequency and amplitude of peaks for each ROI, sample files were unblinded, and statistical comparisons were performed between Control and tbph RNAi groups using a two-tailed unpaired t-test with unequal variance.

\section{$\underline{\text { Analysis of EAAT1 levels on astrocyte membranes }}$}

Data were acquired with a voxel size of $.099 \times .099 \times .23 \mu \mathrm{m}^{3}$ and imported into Fiji. For each sample, raw integrated density (sum of fluorescence intensity unites) was measured from a standard ROI covering $1313 \mu \mathrm{m}$ of the neuropil in the anterior ventral nerve cord. We measured raw integrated density for both EAAT1 and mcd8::GFP (astrocyte membrane marker) from four independent z planes per sample. Finally, we normalized EAAT1 expression to mcd8::GFP expression to account for any potential impacts of tbph knockdown of astrocyte membrane elaboration (Figure 5). 
$\underline{\text { Analysis of synaptic boutons in larval fillet preparations }}$

Data were acquired from dorsal muscles only (DO1-DO2, DA1-DA2) with a voxel size of $.198 \times .198 \times .3 \mu \mathrm{m}^{3}$. Samples were blinded and imported into Fiji, where we performed a max intensity projection of the channel corresponding to vglut-lexA, lexAop-myr::GFP (labels all motor neuron projections and synaptic boutons). Synaptic boutons were quantified manually using the Fiji "Multipoint" tool. After quantifying bouton numbers for an entire experimental set, sample files were unblinded and statistical comparisons were performed using a two-tailed unpaired t-test with unequal variance.

\section{$\underline{\text { Statistical analyses }}$}

Statistics were performed using a combination of Microsoft Excel, MATLAB (MathWorks), and Prism (GraphPad) software. We used a combination of one-way ANOVA, Student's T-Tests (two-tailed, unequal variance), and Mann-Whitney Tests where appropriate (noted throughout). Error bars, Standard Error of the Mean unless otherwise noted. A 95\% confidence interval was used to define the level of significance. Significance: *, p<.05;**, p<.01; ***, p<.001; ****, $\mathrm{p}<.0001, \mathrm{NS}=$ not significant. All other pertinent information, including sample size, statistical test employed, and variance can be found in the figure legends or labeled within the figure. 


\section{METHOD REFERENCES}

57. Crisp, S., Evers, J. F., Fiala, A. \& Bate, M. The development of motor coordination in Drosophila embryos. Dev. Camb. Engl. 135, 3707-3717 (2008).

58. Mauss, A. S., Busch, C. \& Borst, A. Optogenetic Neuronal Silencing in Drosophila during Visual Processing. Sci. Rep. 7, 13823 (2017).

59. Doherty, J., Logan, M. A., Taşdemir, O. E. \& Freeman, M. R. Ensheathing glia function as phagocytes in the adult Drosophila brain. J. Neurosci. Off. J. Soc. Neurosci. 29, 4768-4781 (2009).

60. Peco, E. et al. Drosophila astrocytes cover specific territories of the CNS neuropil and are instructed to differentiate by Prospero, a key effector of Notch. Dev. Camb. Engl. 143, 11701181 (2016).

61. Ingólfsson, H. I. et al. Computational Lipidomics of the Neuronal Plasma Membrane. Biophys. J. 113, 2271-2280 (2017).

62. Perkins, G. et al. Electron tomography of neuronal mitochondria: three-dimensional structure and organization of cristae and membrane contacts. J. Struct. Biol. 119, 260-272 (1997).

63. Artimovich, E., Jackson, R. K., Kilander, M. B. C., Lin, Y.-C. \& Nestor, M. W. PeakCaller: an automated graphical interface for the quantification of intracellular calcium obtained by high-content screening. BMC Neurosci. 18, 72 (2017). 


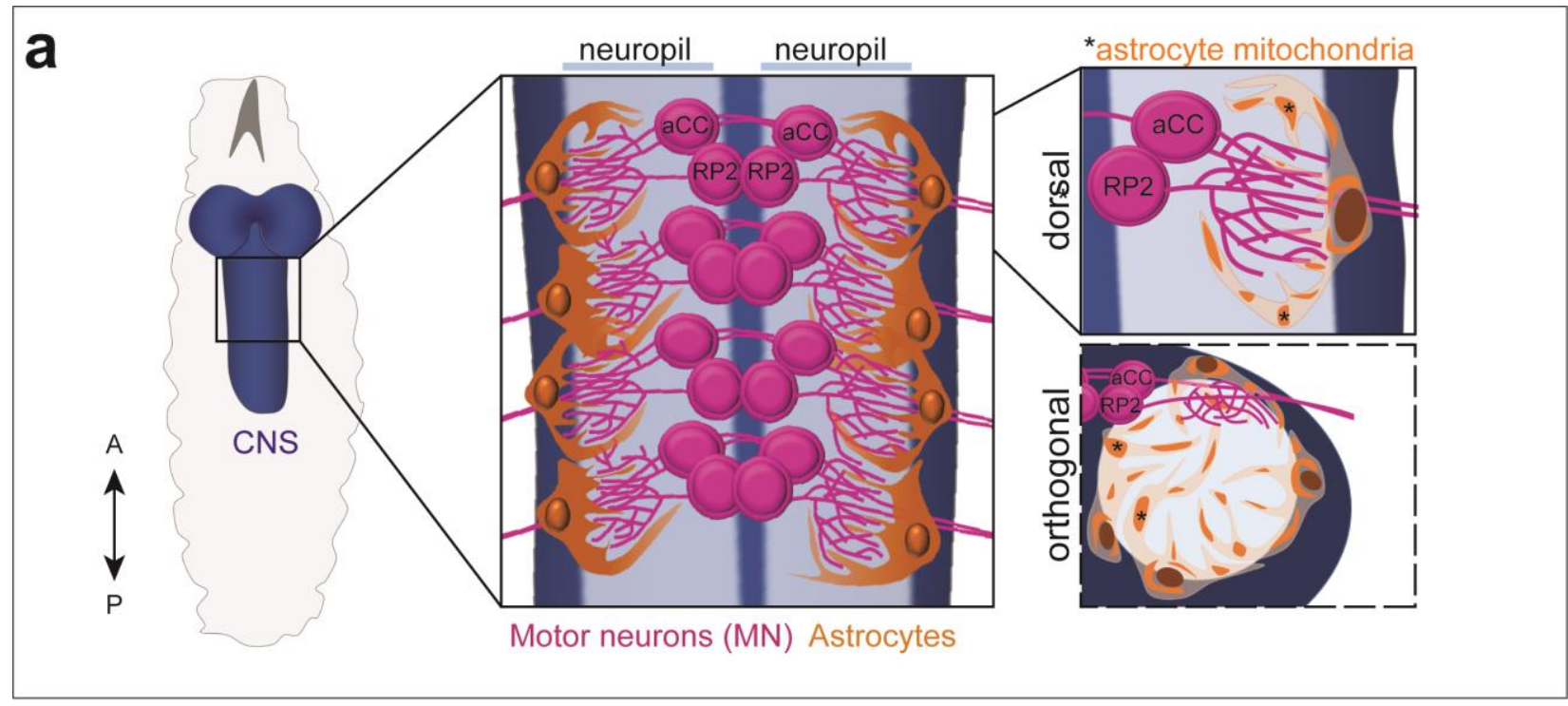

Analysis pipeline: motor synapses associted with astrocyte mitochondria

\section{b Acquire image}

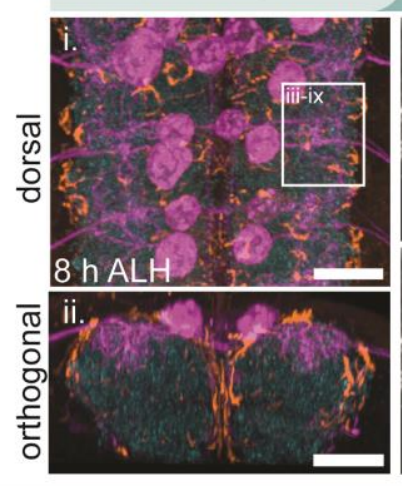

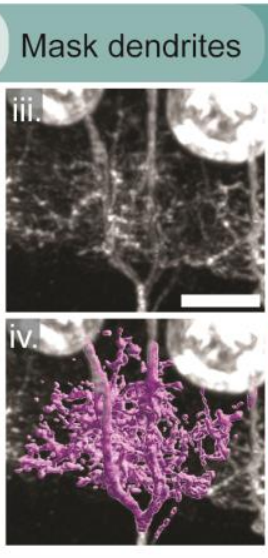

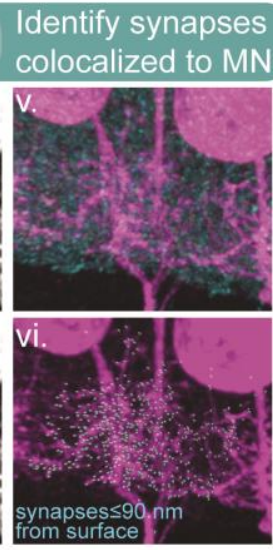

Mask astrocyte
mitochondria
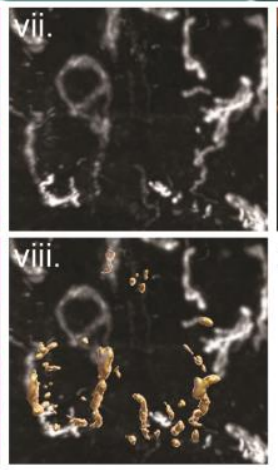

Identify synapses colocalized to mitos.

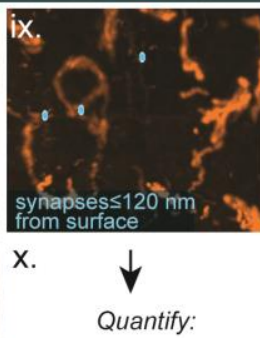

$\%$ mito-assoc synapses mitochondria volume total dendrite volume

Figure 1. Mapping astrocyte-motor neuron, mitochondria-synapse associations in

\section{Drosophila.}

(a) Schematic for reader orientation. A, anterior. P, posterior. Astrocyte mitochondria (orange) are distributed throughout the synapse-rich neuropil (cyan) near motor dendrites (magenta).

(b) Pipeline for identifying motor synapses in close proximity to astrocyte mitochondria at 8 hours after larval hatching (h ALH). (i-ii) Acquire confocal z-stack through neuropil with fluorescently labeled astrocyte mitochondria (R25H07-gal4, UAS-mito::GFP or alrm-lexA, 
lexAop-mcherry::mito.OMM), aCC/RP2 motor neurons (MN; RN2-lexA, lexAop-TdTomato or RN2-gal4, UAS-myr::GFP), and presynapses (Brp+). Scale bar, $10 \mu \mathrm{m}$. (iii-iv) Mask the motor dendrites with an Imaris "Surface." (v-vi) Identify presynapses colocalized with motor dendrites $(\leq 90 \mathrm{~nm})$ and generate Imaris "Spots". (vii-viii) Mask astrocyte mitochondria with an Imaris "Surface." (ix-x) Identify Spots within $120 \mathrm{~nm}$ of masked mitochondria. (iii-ix) Scale bar, $5 \mu \mathrm{m}$. For more details, see Methods. 
MN activation recruits astrocyte mitochondria to dendrites \& synapses
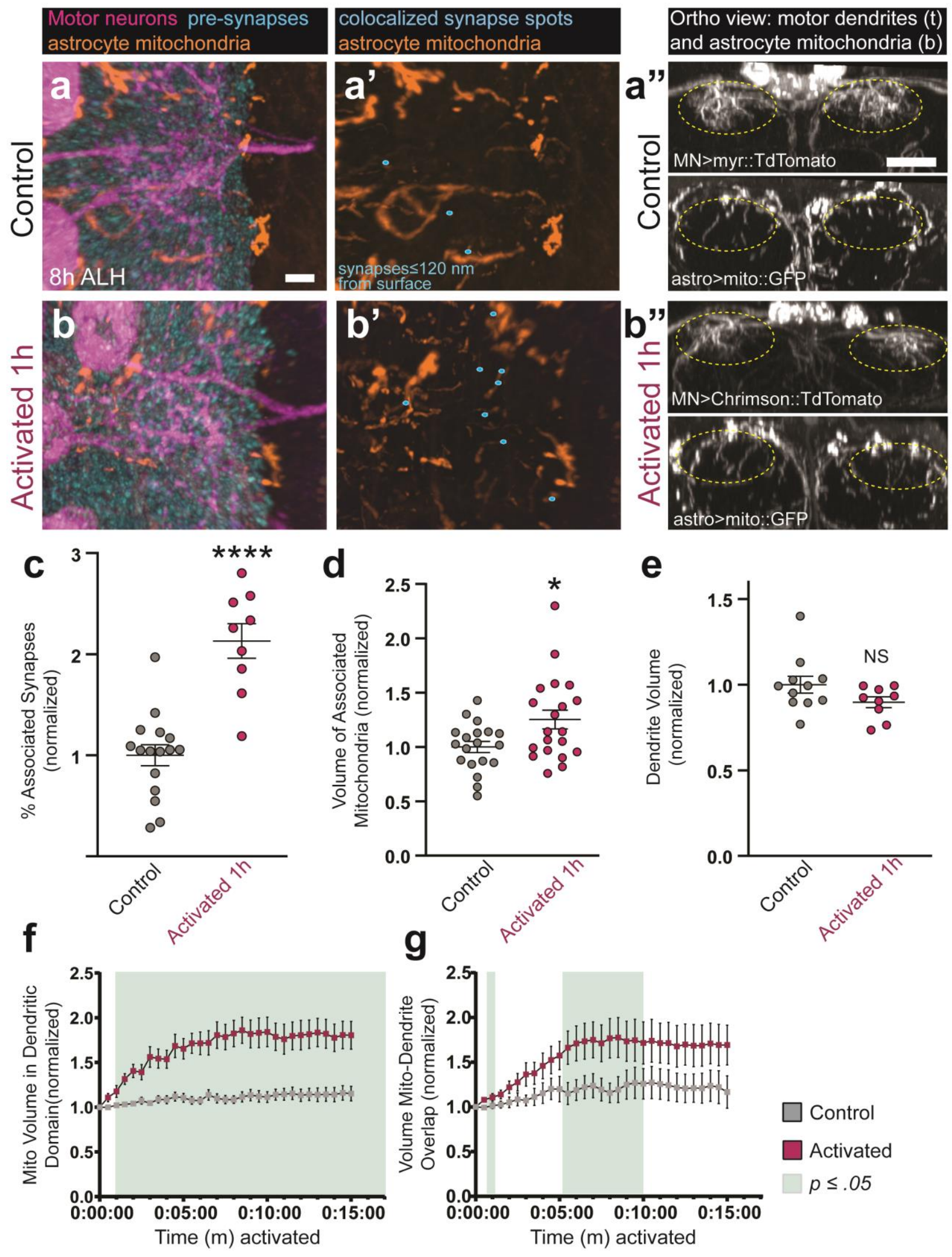
Figure 2. Activation of MNs recruits astrocyte mitochondria to motor dendrites and synapses.

(a-b") Representative images of dark-reared control (a-a") and 1-hour activated (b-b") motor dendrites (magenta, RN2-lexA, lexAop-Chrimson::TdTomato) with neighboring astrocyte synapses (orange, R25H07-gal4, UAS-mito::GFP) and presynapses (cyan, Brp+) in fixed preparations at $8 \mathrm{~h}$ ALH. Prime panels show synapse "Spots" co-localized $(\leq 120 \mathrm{~nm})$ with astrocyte mitochondria. Double prime panels show orthogonal views of motor dendrites (top) and astrocyte mitochondria (bottom) within the dendritic domain (yellow circle). (a-a', b-b') Scale bar, $2 \mu \mathrm{m}$. (a",b") Scale bar, $10 \mu \mathrm{m}$.

(c) Quantification of the percent of synapses associated with astrocyte mitochondria $(\mathrm{p}<.0001$, Mann-Whitney test). Control: $n=16$ animals. Activated: $n=9$ animals. Error bars $=$ SEM.

(d) Quantification of the mitochondrial volume in the dendritic domain ( $\mathrm{p}=.044$, Mann-Whitney test). Control: $n=19$ animals. Activated: $n=20$ animals. Error bars $=$ SEM.

(e) Quantification of dendrite volume ( $\mathrm{p}=.14$, Mann-Whitney test). Control: $\mathrm{n}=11$ animals. Activated: $\mathrm{n}=9$ animals. Error bars $=$ SEM. NS, not significant.

(c-e) $\mathrm{N}$ values reflect animals from 3 technical replicates.

(f-g) Quantification of (f) astrocyte mitochondrial volume (R25H07-gal4, UAS-mito::GFP) within the dendritic domain and (g) overlapping mitochondria-dendrite volume in live samples $(8 \mathrm{~h}$ ALH) imaged every 30 seconds for a 15-minute window of activation (pink, RN2-lexA, lexAopChrimson::TdTomato) or control (grey, RN2-lexA, lexAop-myr::TdTomato). Control: $\mathrm{n}=14$ animals. Activation: $\mathrm{n}=15$ animals. Green areas denote timepoints when $\mathrm{p}<.05$ by one-way ANOVA. Error bars = SEM. N values reflect animals from 2 technical replicates. 


\section{MN silencing repels astrocyte mitochondria from dendrites}
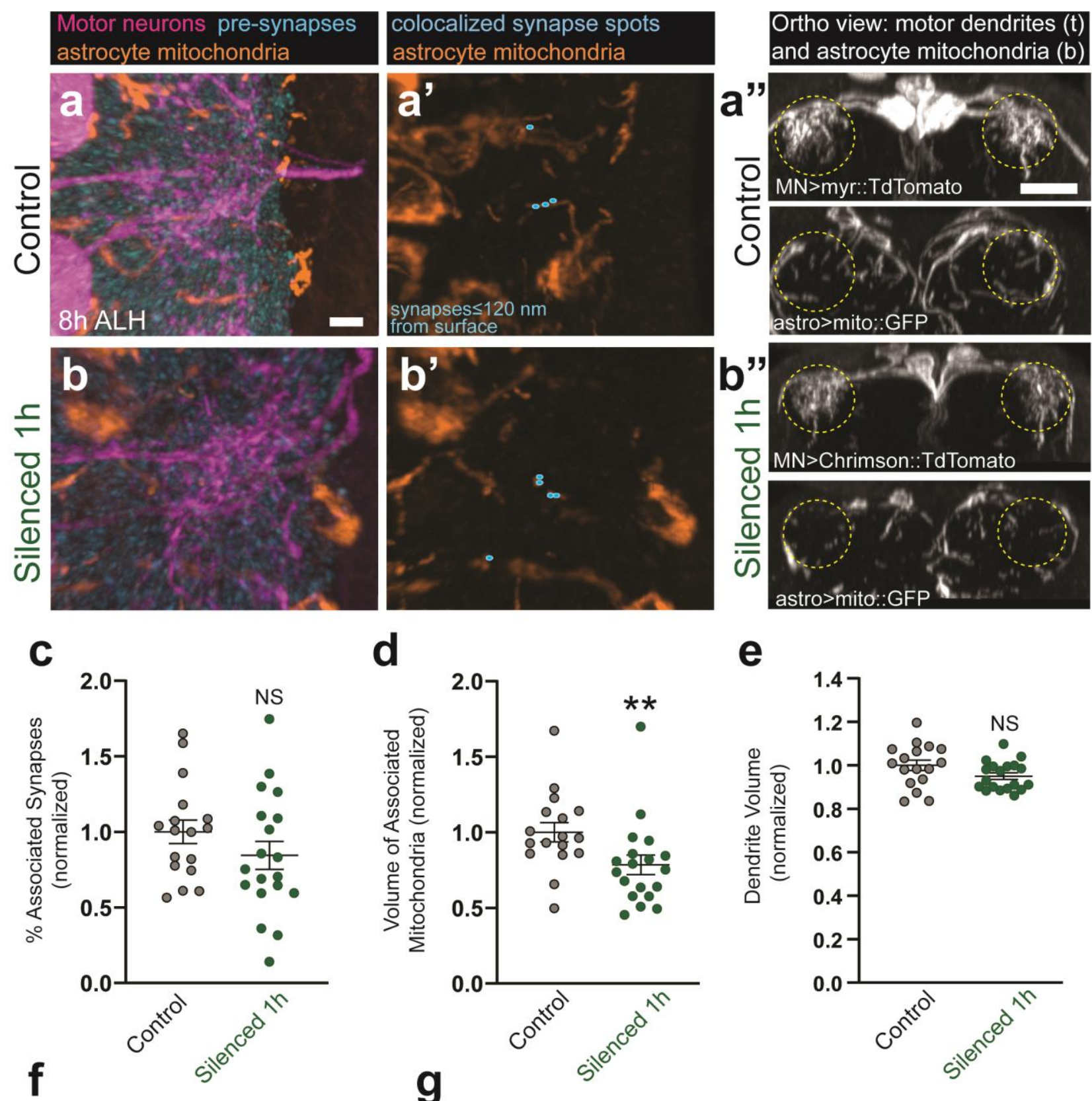

d

e
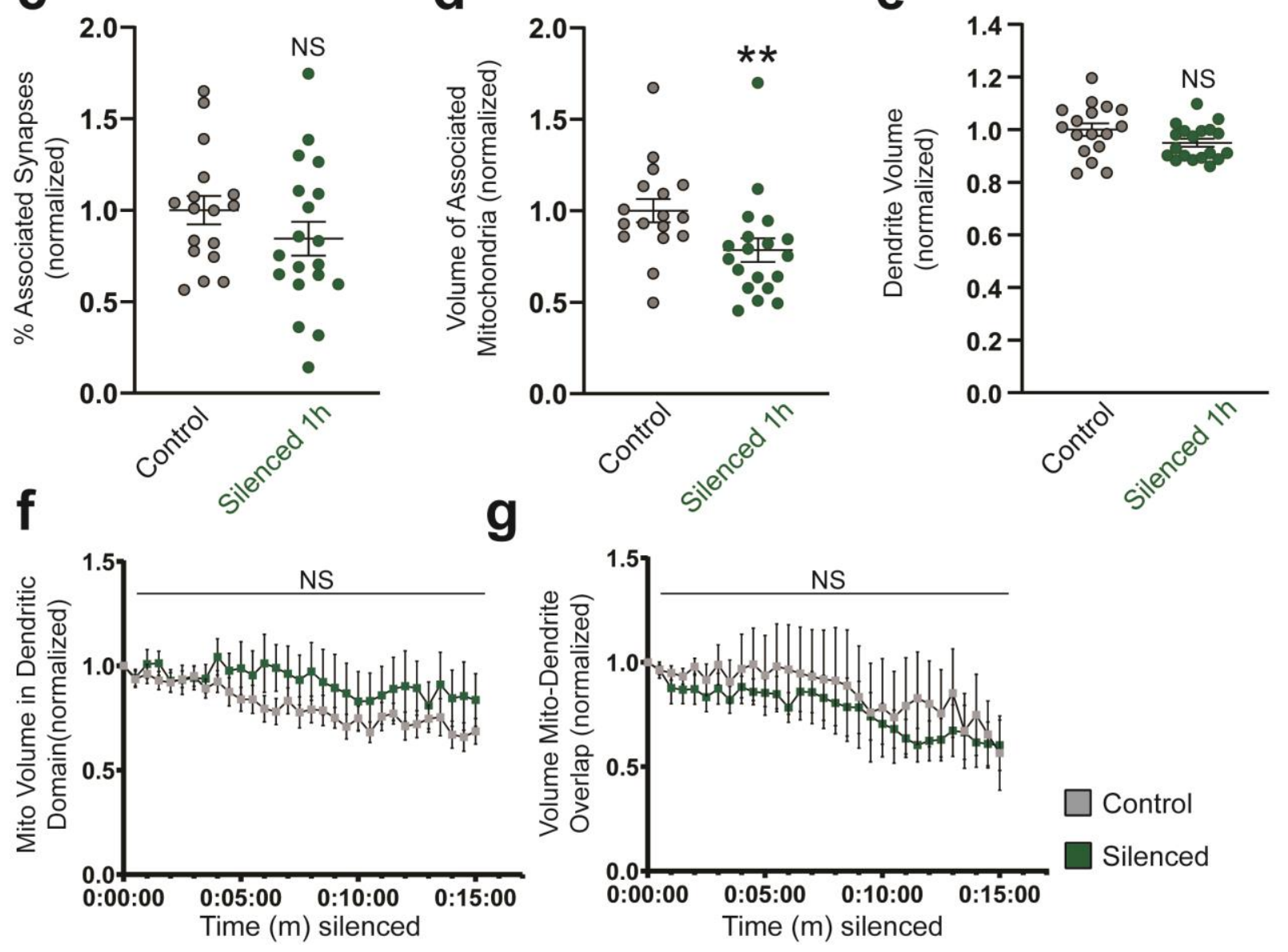


\section{Figure 3. Silencing of MNs repels astrocyte mitochondria from motor dendrites.}

(a-b") Representative images of dark-reared control (a-a") and 1-hour silenced (b-b") motor dendrites (magenta, RN2-gal4, UAS-GtACR2::EYFP) with neighboring astrocyte synapses (orange, alrm-lexA, lexAop-mcherry::mito.OMM) and presynapses (cyan, Brp+) in fixed preparations at $8 \mathrm{~h}$ ALH. Prime panels show synapse "Spots" co-localized $(\leq 120 \mathrm{~nm})$ with astrocyte mitochondria. Double prime panels show orthogonal views of motor dendrites (top) and astrocyte mitochondria (bottom) within the dendritic domain (yellow circle). (a-a', b-b') Scale bar, $2 \mu \mathrm{m}$. (a",b") Scale bar, $10 \mu \mathrm{m}$.

(c) Quantification of the percent of synapses associated with astrocyte mitochondria ( $\mathrm{p}=.26$, Mann-Whitney test). Control: $n=17$ animals. Silenced: $n=19$ animals. Error bars $=$ SEM. NS, not significant.

(d) Quantification of the mitochondrial volume in the dendritic domain ( $\mathrm{p}=.003$, Mann-Whitney test). Control: $n=17$ animals. Silenced: $n=19$ animals. Error bars $=$ SEM.

(e) Quantification of dendrite volume ( $\mathrm{p}=.1$, Mann-Whitney test). Control: $\mathrm{n}=17$ animals.

Silenced: $\mathrm{n}=19$ animals. Error bars $=$ SEM.

(c-e) $\mathrm{N}$ values reflect animals from 3 technical replicates.

(f-g) Quantification of (f) astrocyte mitochondrial volume (alrm-lexA, lexAopmcherry::mito.OMM) within the dendritic domain and (g) overlapping mitochondria-dendrite volume in live samples ( $8 \mathrm{~h} \mathrm{ALH}$ ) imaged every 30 seconds for a 15-minute window of silencing (green, RN2-gal4, UAS-GtACR2::EYFP) or control (grey, RN2-gal4, UAS-myr::GFP). Control: $\mathrm{n}=11$ animals. Silencing: $\mathrm{n}=9$ animals. Error bars $=$ SEM. $\mathrm{N}$ values reflect animals from 2 technical replicates. 


\section{Astrocyte knockdown of tbph enhances mitochondrial trafficking}
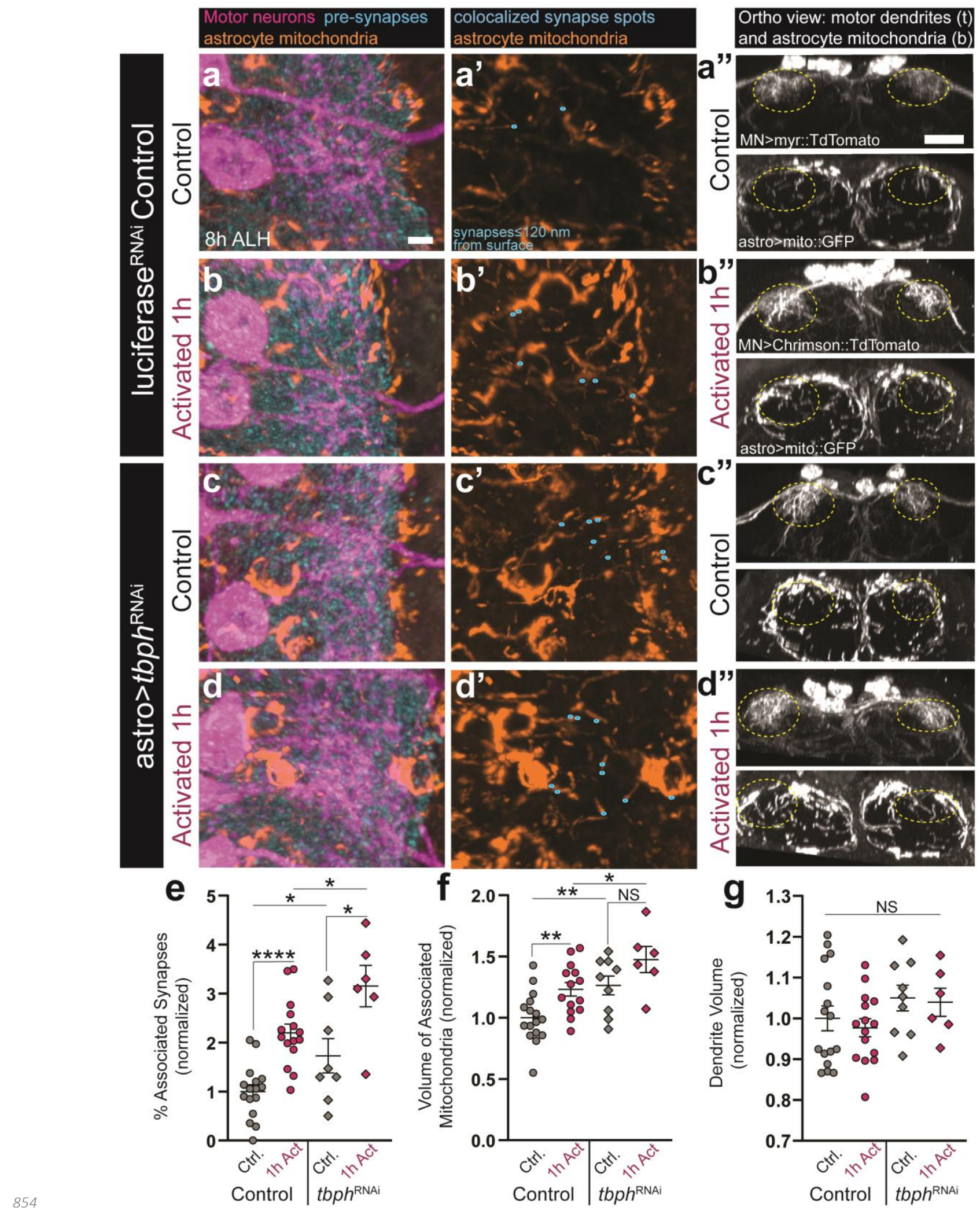
Figure 4. Astrocyte knockdown of tbph enhances activity-dependent mitochondria-synapse association.

(a-b") Representative images of dark-reared control (a-a") and 1-hour activated (b-b") motor dendrites (magenta, RN2-lexA, lexAop-Chrimson::TdTomato) with neighboring astrocyte synapses (orange, R25H07-gal4, UAS-mito::GFP) and presynapses (cyan, Brp+) in fixed preparations of control (UAS-luciferase $\left.{ }^{\mathrm{RNAi}}\right)$ animals at $8 \mathrm{~h}$ ALH. Prime panels show synapse "Spots" co-localized $(\leq 120 \mathrm{~nm})$ with astrocyte mitochondria. Double prime panels show orthogonal views of motor dendrites (top) and astrocyte mitochondria (bottom) within the dendritic domain (yellow circle). (a-a', b-b') Scale bar, $2 \mu \mathrm{m}$. (a",b") Scale bar, $10 \mu \mathrm{m}$. (c-d") Representative images of dark-reared control (a-a") and 1-hour activated (b-b") motor dendrites (magenta, RN2-lexA, lexAop-Chrimson::TdTomato) with neighboring astrocyte synapses (orange, R25H07-gal4, UAS-mito::GFP) and presynapses (cyan, Brp+) in fixed preparations of astrocyte $t b p h$ knockdown $\left(U A S-t b p h^{\mathrm{RNAi}}\right)$ animals at $8 \mathrm{~h}$ ALH. Prime panels show synapse "Spots" co-localized $(\leq 120 \mathrm{~nm})$ with astrocyte mitochondria. Double prime panels show orthogonal views of motor dendrites (top) and astrocyte mitochondria (bottom) within the dendritic domain (yellow circle). (c-c', d-d') Scale bar, $2 \mu \mathrm{m}$. (c”,d”) Scale bar, $10 \mu \mathrm{m}$.

(e) Quantification of the percent of synapses associated with astrocyte mitochondria. Statistical significance quantified by one-way ANOVA: dark-reared versus activated control $(\mathrm{p}<.0001)$; dark-reared versus activated $t b p h^{\mathrm{RNAi}}(\mathrm{p}<.03)$; dark-reared comparisons ( $\left.\mathrm{p}<.03\right)$; activated comparisons $(\mathrm{p}<.03)$.

(f) Quantification of mitochondrial volume in the dendritic domain. Statistical significance quantified by one-way ANOVA: dark-reared versus activated control ( $\mathrm{p}<.005)$; dark-reared 
877 versus activated $t b p h^{\mathrm{RNAi}}(\mathrm{p}<.13)$; dark-reared comparisons $(\mathrm{p}<.008)$; activated comparisons

$878 \quad(\mathrm{p}<.04)$. NS, not significant.

879

880

881

882

883

884

885

886

887 (g) Quantification of dendrite volume. Statistical significance quantified by one-way ANOVA:

dark-reared versus activated control $(\mathrm{p}<.55)$; dark-reared versus activated $t b p h^{\mathrm{RNAi}}(\mathrm{p}<.84)$; darkreared comparisons $(\mathrm{p}<.3)$; activated comparisons $(\mathrm{p}<.15)$.

(e-g) Dark-reared control: $\mathrm{n}=16$ animals. Activated control: $\mathrm{n}=15$ animals. Dark-reared $t b p h^{\mathrm{RNAi}}$ : $\mathrm{n}=8$ animals. Activated $t b p h^{\mathrm{RNAi}}: \mathrm{n}=6$ animals. Error bars $=\mathrm{SEM} . \mathrm{N}$ values reflect animals from 3 technical replicates. 
Astrocyte $t b p h^{\mathrm{RNAi}}$ increases frequency and amplitude of MN activity
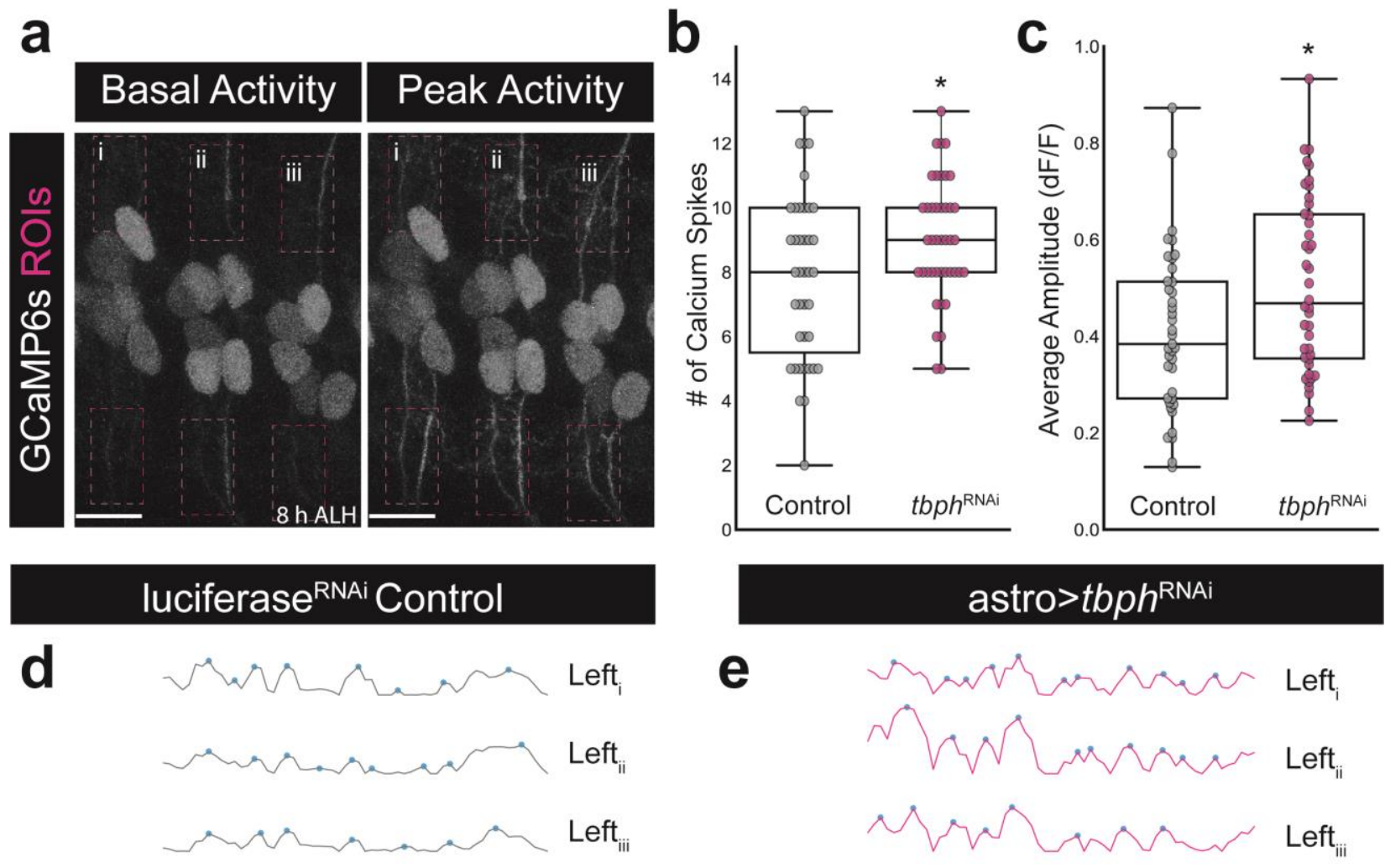

Right $_{\mathrm{i}}$

Right $_{\mathrm{i}}$
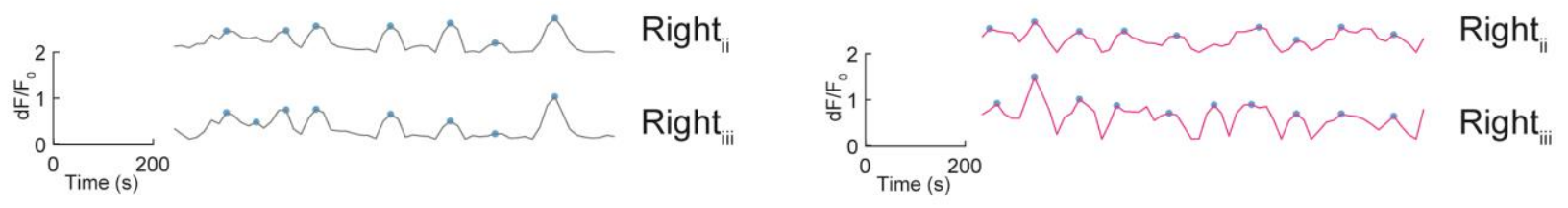

Knockdown of tbph causes significant reduction in astrocyte EAAT1

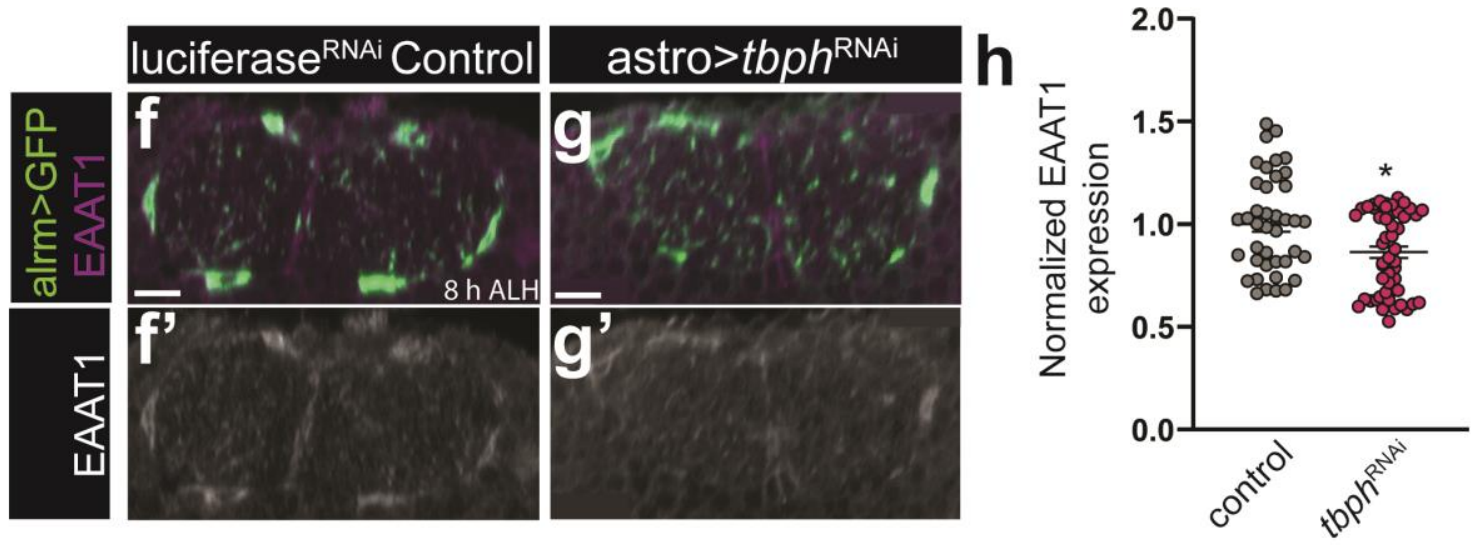




\section{Figure 5. Astrocyte tbph knockdown increases frequency and amplitude of motor neuron} activity.

(a) Maximum intensity projection of GCaMP7s activity in aCC/RP2 (RN2-lexA, lexAopGCaMP7s) in control at basal (left) and peak (right) activity at $8 \mathrm{~h} \mathrm{ALH.} \mathrm{Anterior} \mathrm{to} \mathrm{the} \mathrm{left,}$ dorsal is up. Pink boxes are ROIs used for $\mathrm{dF} / \mathrm{F}$ quantification.

(b) Frequency of calcium peak events for control (gray, R25H07-gal4, UAS-luciferase ${ }^{\mathrm{RNAi}}$ ) and tbph knockdown (pink, R25H07-gal4, UAS-tbph ${ }^{\mathrm{RNAi}}$ ) animals. $t b p h^{R N A i}$ animals exhibited a higher frequency of calcium peaks (Student's t-test, $\mathrm{p}=0.023$ ). Each point represents an individual hemisegment. Control: $\mathrm{n}=39$ hemisegments from 7 animals, $t b p h^{R N A i}: \mathrm{n}=42$ hemisegments from 7 animals. Error bars $=$ SD.

(c) Average dF/F amplitude of calcium peaks for control (gray) and $t b p h^{R N A i}$ (pink) animals. $t b p h^{R N A i}$ animals exhibited an increase in calcium peak amplitude (Student's t-test, $\mathrm{p}=0.013$ ). Each point represents an individual hemisegment. Control: $\mathrm{n}=39$ hemisegments from 7 animals, $t b p h^{R N A i}: \mathrm{n}=42$ hemisegments from 7 animals. Error bars $=\mathrm{SD}$.

(b-c) $\mathrm{N}$ values reflect animals from 3 technical replicates.

(d-e) Representative $\mathrm{dF} / \mathrm{F}$ traces from a single control (d) and $t b p h^{R N A i}(\mathbf{e})$ animal. Each trace is from an individual hemisegment. Hemisegment position (Left/Right, Anterior(i)-Posterior(iii)) indicated to the right of each trace. Peaks of calcium activity identified by PeakCaller (see Methods) are shown as blue dots in each trace.

(f-g) Orthogonal view showing EAAT1 expression in (f) control astrocytes (alrm-gal4, UAS$m c d 8 G F P)$ or $(\mathbf{g})$ astrocytes following tbph RNAi knockdown $\left(U A S\right.$-tbph $\left.{ }^{\mathrm{RNAi}}\right)$ at $8 \mathrm{~h}$ ALH. Prime panels show EAAT1 staining alone. Control: $\mathrm{n}=40$ sections from 10 animals, $t b p h^{\mathrm{RNAi}}$ : $\mathrm{n}=52$ segments from 13 animals. $\mathrm{N}$ values reflect animals from 2 technical replicates. 
bioRxiv preprint doi: https://doi.org/10.1101/2021.11.29.470476; this version posted December 1, 2021. The copyright holder for this preprint (which was not certified by peer review) is the author/funder. All rights reserved. No reuse allowed without permission.

(h) Quantification of EAAT1 expression relative to membrane bound GFP on astrocyte membranes ( $\mathrm{p}=.018$, Mann-Whitney test). Error bars $=$ SEM. 


\section{Identification of molecular machinery for astrocyte mitochondrial trafficking}
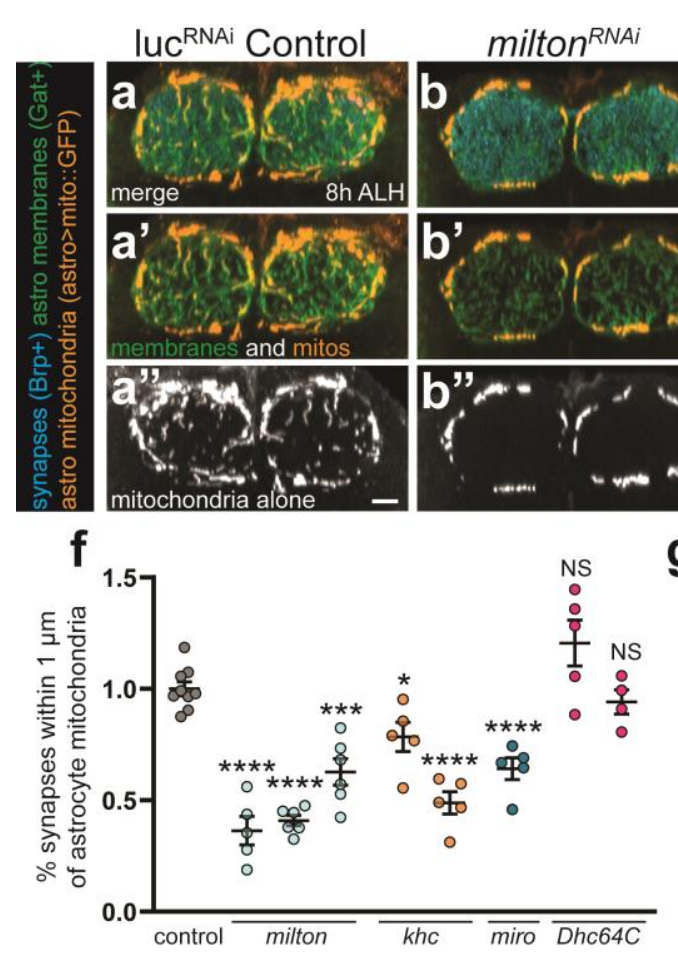

milton $^{R N A i}$
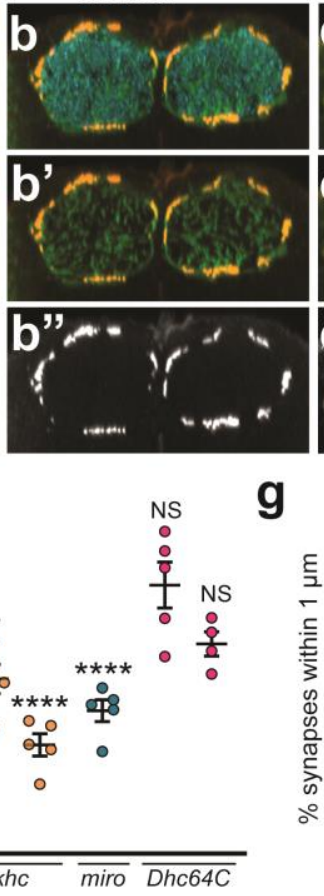
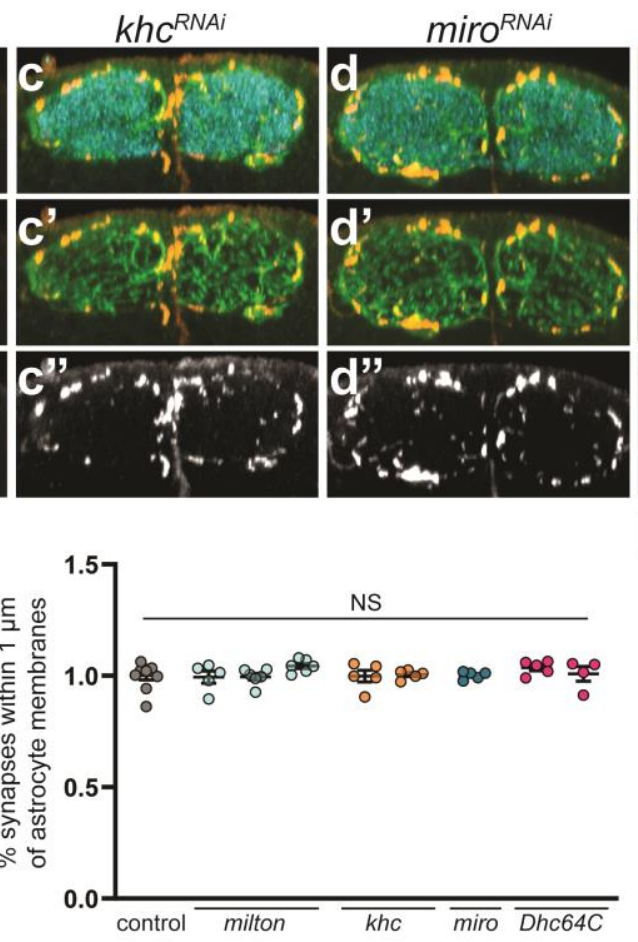

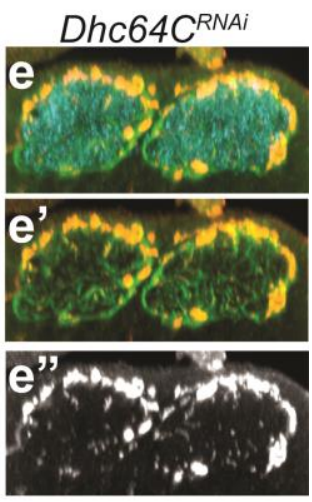

h

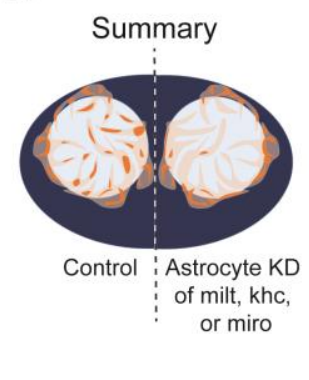

Figure 6. Astrocyte mitochondria traffic along (+) ended microtubules.

(a-e") Representative orthogonal views of astrocyte membranes (Gat+, green), mitochondria (R25H07-gal4, UAS-mito::GFP, orange), and neighboring synapses (Brp+, cyan) in (a-a")

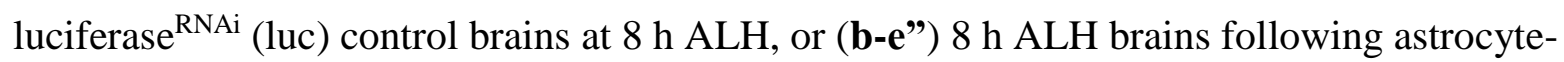
specific knockdown of molecular machinery required for mitochondrial transport in axons.

Prime panels show astrocyte membranes and mitochondria alone. Double prime panels show mitochondria alone. Scale bar, $5 \mu \mathrm{m}$ for all panels.

(f) Quantification of the percent of all synapses within $1 \mu \mathrm{m}$ of astrocyte mitochondria.

Statistical significance compared to control determined by one-way ANOVA: milton $^{\text {RNAi } 1}$ $(\mathrm{p}<.0001)$, milton $^{\mathrm{RNAi} 2}(\mathrm{p}<.0001)$, milton $^{\mathrm{RNAi} 3}(\mathrm{p}<.002), k h c^{\mathrm{RNAi} 1}(\mathrm{p}<.04), k h c^{\mathrm{RNAi} 2}(\mathrm{p}<.007)$, miro $^{\mathrm{RNAi}}(\mathrm{p}<.0001), \operatorname{Dhc} 64 C^{\mathrm{RNAi} 1}(\mathrm{p}<.14), \operatorname{Dhc}^{\mathrm{R}} 64 C^{\mathrm{RNAi} 2}(\mathrm{p}<.43)$. 
(g) Quantification of the percent of all synapses within $1 \mu \mathrm{m}$ of astrocyte membranes.

Statistical significance compared to control determined by one-way ANOVA: ilton $^{\mathrm{RNAi}} 1$ $(\mathrm{p}<.84)$, milton $^{\mathrm{RNAi} 2}(\mathrm{p}<.85)$, milton $^{\mathrm{RNAi} 3}(\mathrm{p}<.24), k h c^{\mathrm{RNAi} 1}(\mathrm{p}<.91), k h c^{\mathrm{RNAi} 2}(\mathrm{p}<.97)$, $\operatorname{miro}^{\mathrm{RNAi}}(\mathrm{p}<.0001), D h c 64 C^{\mathrm{RNAi} 1}(\mathrm{p}<.37), D h c 64 C^{\mathrm{RNAi} 2}(\mathrm{p}<.88)$.

(f-g) Control: $\mathrm{n}=9$. Milton $^{\text {RNAi } 1}: \mathrm{n}=6$. Milton $^{\mathrm{RNAi}}$ 2. $\mathrm{n}=5$. Milton $^{\text {RNAi } 3}: \mathrm{n}=6$. Khc $^{\text {RNAi } 1}: \mathrm{n}=5$. Khe ${ }^{\text {RNAi } 2}: \mathrm{n}=5$ animals. Miro ${ }^{\mathrm{RNAi}}: \mathrm{n}=5$ animals. Dhc $64 C^{\mathrm{RNAi}}{ }^{1}: \mathrm{n}=5$ animals. $D h c 64 C^{\mathrm{RNAi}}{ }^{1}$ : $\mathrm{n}=4$ animals. Error bars $=$ SEM. $\mathrm{N}$ values reflect animals from 2 technical replicates.

(h) Graphical summary of screen results. 


\section{Blocking astrocyte mitochondrial transport modifies locomotor behavic}

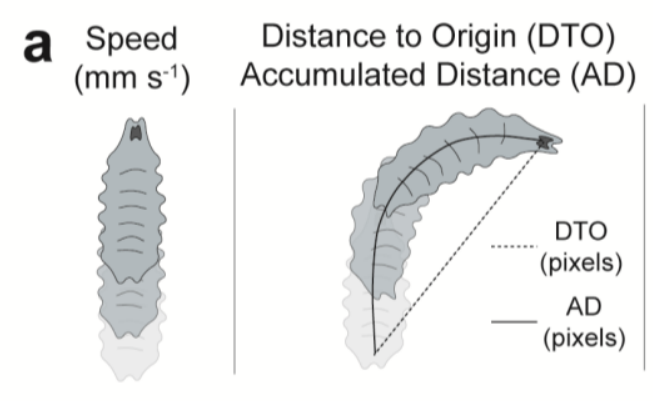
Cumulative Bending
Angle (CBA)

Crawling
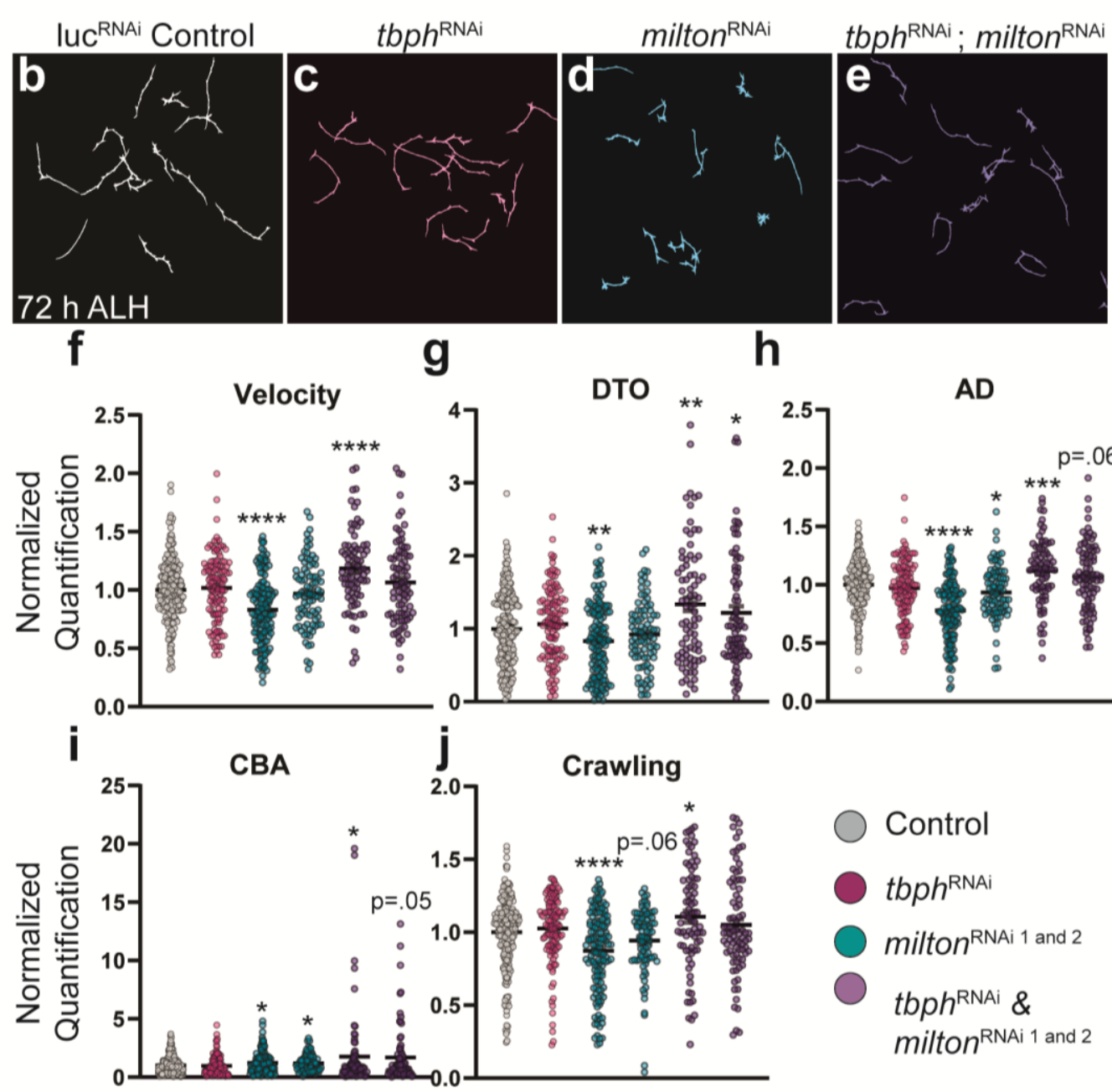

\begin{tabular}{l|c}
$b_{i}=\left|180-\theta_{i}\right|$ & $c_{i}=$ crawling frame \\
CBA $=\Sigma b_{i}$ & Crawling $=\left(\Sigma c_{i}\right) / i * 100$
\end{tabular}

\begin{tabular}{l|c}
$b_{i}=\left|180-\theta_{i}\right|$ & $c_{i}=$ crawling frame \\
CBA $=\Sigma b_{i}$ & Crawling $=\left(\Sigma c_{i}\right) / i{ }^{*} 100$
\end{tabular}

tbph $h^{\mathrm{RNAi}} ;$ milton $^{\mathrm{RNAi}}$

Figure 7. Astrocyte mitochondrial trafficking modifies larval crawling behavior.

937 (a) Schematic of behavioral paradigms tested.

(b-e) FIMTrack traces from individual, free-crawling larvae recorded for 1 minute at $4 \mathrm{~Hz}$.

Luciferase $^{\mathrm{RNAi}}$ (luc) Control: $\mathrm{n}=222$ animals. Tbph $^{\mathrm{RNAi}}: \mathrm{n}=126$ animals. Milton $^{\text {RNAi }}{ }^{1}: \mathrm{n}=139$ 
940

941

942

943

944

945

946

947

948

949

950

951

animals. Milton $^{\mathrm{RNAi} 2}: \mathrm{n}=85$ animals. Tbph $^{\mathrm{RNAi}}+$ milton $^{\mathrm{RNAi}}{ }^{1}: \mathrm{n}=79$ animals. Tbph $^{\mathrm{RNAi}}+$ milton $^{\text {RNAi } 2}: \mathrm{n}=82$ animals. $\mathrm{N}$ values reflect animals from 3 technical replicates.

(f-j) Quantification of behavioral metrics relative to control. Statistical significance determined by Student's T-Test for (f) velocity $\left(t b p h^{\mathrm{RNAi}}, \mathrm{p}<.57 ;\right.$ milton $^{\mathrm{RNAi}}{ }^{1}, \mathrm{p}<.0001$; milton $^{\mathrm{RNAi} 2}, \mathrm{p}<.4 ;$ tbph $^{\mathrm{RNAi}}+$ milton $^{\mathrm{RNAi} 1}, \mathrm{p}<.0001 ;$ tbph $^{\mathrm{RNAi}}+$ milton $\left.^{\mathrm{RNAi} 2}, \mathrm{p}<.14\right),(\mathrm{g})$ distance to origin $\left(t b p h^{\mathrm{RNAi}}, \mathrm{p}<.26 ;\right.$ milton $^{\mathrm{RNAi} 1}, \mathrm{p}<.003 ;$ milton $^{\mathrm{RNAi} 2}, \mathrm{p}<.21 ; t^{2} p h^{\mathrm{RNAi}}+$ milton $^{\mathrm{RNAi} 1}, \mathrm{p}<.002 ;$ tbph $^{\mathrm{RNAi}}+$ milton $\left.^{\mathrm{RNAi} 2}, \mathrm{p}<.03\right)$, (h) accumulated distance $\left(t b p h^{\mathrm{RNAi}}\right.$, $\mathrm{p}<.26 ;$ milton $^{\mathrm{RNAi} 1}, \mathrm{p}<.0001 ;$ milton $^{\mathrm{RNAi} 2}, \mathrm{p}<.03 ;$ tbph $^{\mathrm{RNAi}}+$ milton $^{\mathrm{RNAi} 1}, \mathrm{p}<.0001 ;$ tbph $^{\mathrm{RNAi}}+$ milton $\left.^{\mathrm{RNAi} 2}, \mathrm{p}<.06\right)$, (i) cumulative bending angle (tbph ${ }^{\mathrm{RNAi}}, \mathrm{p}<.68 ;$ milton $^{\mathrm{RNAi} 1}, \mathrm{p}<.03$; milton $^{\mathrm{RNAi} 2}, \mathrm{p}<.05 ;$ tbph $^{\mathrm{RNAi}}+$ milton $^{\mathrm{RNAi} 1}, \mathrm{p}<.05 ;$ tbph $^{\mathrm{RNAi}}+$ milton $\left.^{\mathrm{RNAi} 2}, \mathrm{p}=.06\right)$, and $(\mathbf{j})$ crawling $\left(\right.$ tbph $^{\mathrm{RNAi}}, \mathrm{p}<.33 ;$ milton $^{\mathrm{RNAi} 1}, \mathrm{p}<.0001 ;$ milton $^{\mathrm{RNAi} 2}, \mathrm{p}=.05 ;$ tbph $^{\mathrm{RNAi}}+$ milton $^{\mathrm{RNAi}}{ }^{1}$, $\mathrm{p}<.02 ;$ tbph $^{\mathrm{RNAi}}+$ milton $\left.^{\mathrm{RNAi} 2}, \mathrm{p}<.22\right)$. Error bars $=\mathrm{SEM}$. 

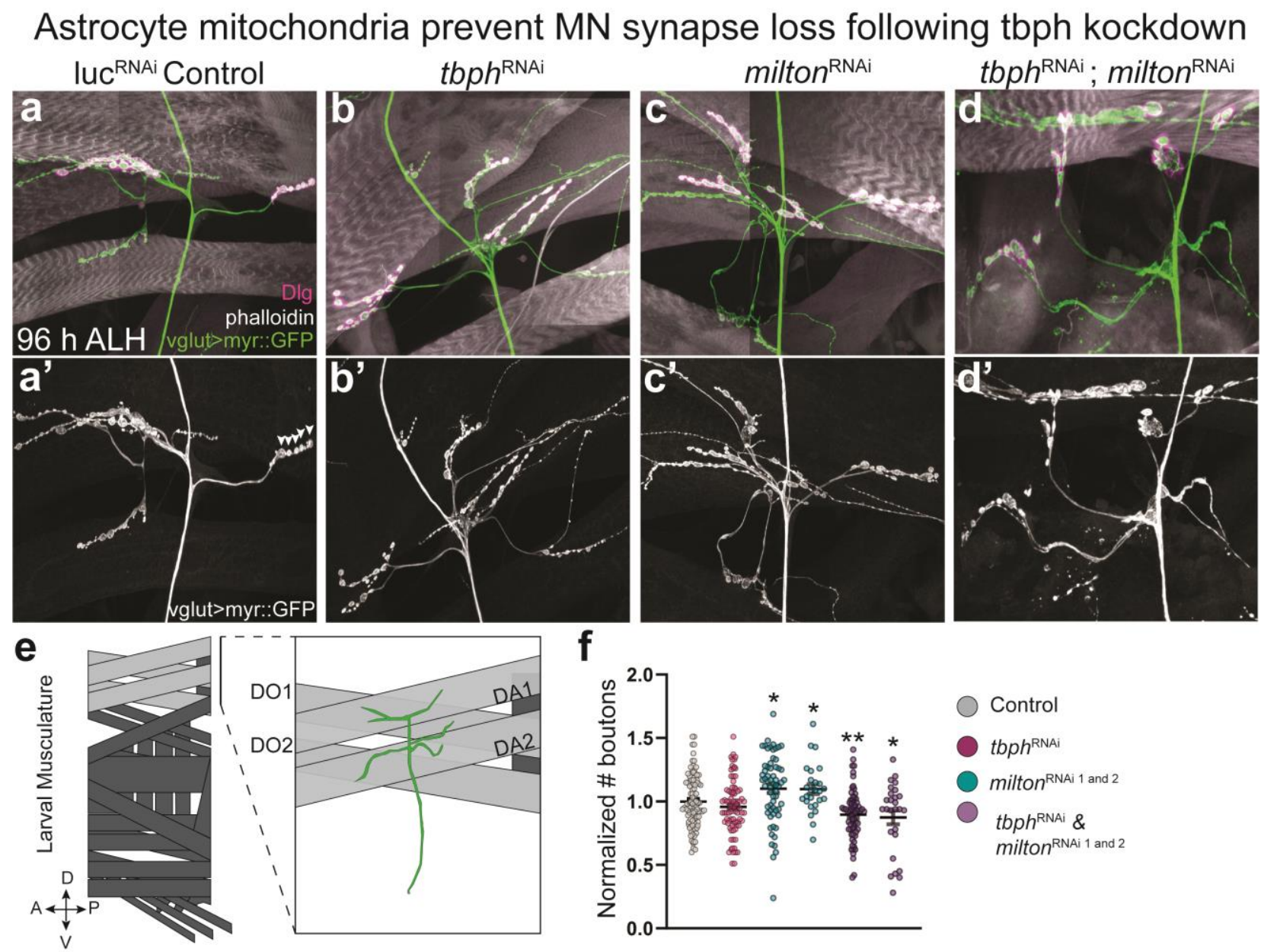

Control

$t b p h^{\mathrm{RNAi}}$

milton $^{\mathrm{RNAi} 1}$ and 2

tbph RNAi \& milton ${ }^{\text {RNAi } 1 \text { and } 2}$

Figure 8. Peri-synaptic astrocyte mitochondria are necessary for synapse maintenance.

(a-d') Representative images of $96 \mathrm{~h}$ ALH neuromuscular junctions (NMJs, Green: vglutlexA, lexAop-myr::GFP; Magenta: Dlg+; White: Phalloidin) from luciferase ${ }^{\mathrm{RNAi}}$ (luc) control (a) or following astrocyte-specific knockdown of (b) tbph, (c) milton, or (d) both tbph and milton together (R25H07-gal4, UAS-RNAi). Prime panels show GFP channel used for quantification of synaptic boutons (arrowheads). Control: $n=98$ NMJs from $n=16$ animals. Tbph $^{\text {RNAi }}: \mathrm{n}=81$ NMJs from $\mathrm{n}=14$ animals. Milton $^{\mathrm{RNAi}}$ : $\mathrm{n}=61$ NMJs from $\mathrm{n}=12$ animals. Milton $^{\text {RNAi } 2}: \mathrm{n}=25$ NMJs from $\mathrm{n}=4$ animals. Tbph $^{\text {RNAi }}+$ milton $^{\text {RNAi } 1}: \mathrm{n}=64$ NMJs from $\mathrm{n}=12$ animals. Tbph $^{\mathrm{RNAi}}+$ milton $^{\mathrm{RNAi}}$ : $\mathrm{n}=26 \mathrm{NMJ}$ from $\mathrm{n}=4$ animals. $\mathrm{N}$ values reflect animals from 2 technical replicates. 
964

965

966

967

968

969

970

971

(e) Schematic for reader orientation.

(f) Quantification of bouton number (type 1b only). Statistical significance determined by

Student's T-Test relative to control: $t_{b p h} h^{\mathrm{RNAi}}(\mathrm{p}<.19)$, milton $^{\mathrm{RNAi} 1}(\mathrm{p}<.02)$, milton $^{\mathrm{RNAi} 2}$

$(\mathrm{p}<.03)$, tbph $^{\mathrm{RNAi}}+$ milton $^{\mathrm{RNAi} 1}(\mathrm{p}<.002)$, tbph $^{\mathrm{RNAi}}+$ milton $^{\mathrm{RNAi} 2}(\mathrm{p}<.04)$. 


\section{SUPPLEMENTAL MOVIE LEGENDS}

973

Supplementary Movies 1-11: All movies are .mov files and can be visualized using standard media players.

\section{Supplementary Movie 1. Time-lapse imaging of astrocyte mitochondria in myr::TdTomato} control for Chrimson-activation.

Representative time-lapse movie showing astrocyte mitochondrial dynamics (R25HO7-gal4, UAS-mito::GFP, green) around aCC/RP2 dendrites (RN2-lexA, lexAop-myr::TdTomato) in a fictive brain preparation at $8 \mathrm{~h} \mathrm{ALH}$. Animals were supplied with ATR and reared in the dark to mimic conditions for Chrimson-activation. A single z-stack was acquired every 30 seconds (each stack taking 25 " to acquire) and brains were imaged for a total of 15 minutes. Astrocyte mitochondria were highly motile, though largely stable in volume over the 15-minute period. Left hemisegment shows Imaris "Surface" reconstruction of astrocyte mitochondria, right shows raw data in neighboring hemisegment.

\section{Supplementary Movie 2. Time-lapse imaging of astrocyte mitochondria during motor} neuron Chrimson-activation.

Representative time-lapse movie showing astrocyte mitochondrial dynamics (R25H07-gal4, UAS-mito::GFP, green) around aCC/RP2 dendrites (RN2-lexA, lexAop-Chrimson::TdTomato) in a fictive brain preparation at $8 \mathrm{~h}$ ALH. Animals were supplied with ATR and dark-reared. A single z-stack was acquired every 30 seconds (each stack taking 25 " to acquire) and brains were imaged for a total of 15 minutes. Astrocyte mitochondria were rapidly trafficked towards motor 
dendrites in the first several minutes, followed by relative stability. Left hemisegment shows Imaris "Surface" reconstruction of astrocyte mitochondria, right shows raw data in neighboring hemisegment.

\section{Supplementary Movie 3. Time-lapse imaging of astrocyte mitochondria in myr::GFP control for GtACR2 silencing.} Representative time-lapse movie showing astrocyte mitochondrial dynamics (alrm-lexA, lexAopmcherry::mito.OMM, magenta) around aCC/RP2 dendrites (RN2-gal4, UAS-GtACR2::EYFP) in a fictive brain preparation at $8 \mathrm{~h}$ ALH. Animals were supplied with ATR and reared in the dark to mimic conditions for GtACR2-silencing. A single z-stack was acquired every 30 seconds (each stack taking 25 " to acquire) and brains were imaged for a total of 15 minutes. Astrocyte mitochondria were highly motile, though largely stable in volume over the 15-minute period. Left hemisegment shows Imaris "Surface" reconstruction of astrocyte mitochondria, right shows raw data in neighboring hemisegment.

\section{Supplementary Movie 4. Time-lapse imaging of astrocyte mitochondria during motor neuron GtACR2-silencing.}

Representative time-lapse movie showing astrocyte mitochondrial dynamics (alrm-lexA, lexAopmcherry::mito.OMM, magenta) around aCC/RP2 dendrites (RN2-gal4, UAS-GtACR2::EYFP) in a fictive brain preparation at $8 \mathrm{~h}$ ALH. Animals were supplied with ATR and reared in the dark. A single z-stack was acquired every 30 seconds (each stack taking 25 " to acquire) and brains were imaged for a total of 15 minutes. Astrocyte mitochondria were highly motile, though largely 
stable in volume over the 15-minute period. Left hemisegment shows Imaris "Surface" reconstruction of astrocyte mitochondria, right shows raw data in neighboring hemisegment.

\section{Supplementary Movie 5. Time-lapse imaging of wild-type aCC/RP2 activity.} Representative time-lapse movie showing control aCC/RP2 activity in a fictive brain preparation at $8 \mathrm{~h}$ ALH (RN2-lexA, lexAop-GCaMP7s; R25H07-gal4, UAS-luciferase $\left.{ }^{\mathrm{RNAi}}\right)$. A single z-stack was acquired every 15 seconds (each stack taking 15 " to acquire) and brains were imaged for a total of 15 minutes.

\section{Supplementary Movie 6. Time-lapse imaging of aCC/RP2 activity following astrocyte}

\section{knockdown of $t b p h$.}

Representative time-lapse movie showing aCC/RP2 activity in a fictive brain preparation at $8 \mathrm{~h}$ ALH (RN2-lexA, lexAop-GCaMP7s) following astrocyte knockdown of tbph (R25H07-gal4, $U A S-t b p h^{\mathrm{RNAi}}$ ). A single z-stack was acquired every 15 seconds (each stack taking 15 " to acquire) and brains were imaged for a total of 15 minutes. Activity was elevated relative to control.

\section{Supplementary Movie 7. Time lapse imaging showing control astrocyte mitochondrial} dynamics.

Representative time-lapse movie showing astrocyte mitochondria (R25HO7-gal4, UASmito::GFP) dynamics in a control brain (UAS-luciferase $\left.{ }^{\mathrm{RNAi}}\right)$ at $8 \mathrm{~h}$ ALH. A single $\mathrm{z}$-stack was acquired one a minute (each stack taking 15" to acquire) and brains were imaged for a total of 10 minutes. Mitochondria were distributed throughout the neuropil. 
Supplementary Movie 8. Time lapse imaging showing astrocyte mitochondrial dynamics following astrocyte knockdown of milton.

Representative time-lapse movie showing astrocyte mitochondria (R25H07-gal4, UASmito::GFP) dynamics in a milton knockdown brain $\left(U A S-\right.$ milton $\left.^{\mathrm{RNAi}}\right)$ at $8 \mathrm{~h}$ ALH. A single zstack was acquired one a minute (each stack taking 15" to acquire) and brains were imaged for a total of 10 minutes. Mitochondria were absent from the neuropil.

Supplementary Movie 9. Time lapse imaging showing astrocyte mitochondrial dynamics following astrocyte knockdown of $k h c$.

Representative time-lapse movie showing astrocyte mitochondria (R25H07-gal4, UASmito::GFP) dynamics in a khc knockdown brain $\left(U A S-k h c^{\mathrm{RNAi}}\right)$ at $8 \mathrm{~h}$ ALH. A single z-stack was acquired one a minute (each stack taking 15 " to acquire) and brains were imaged for a total of 10 minutes. Mitochondria were reduced in the neuropil.

\section{Supplementary Movie 10. Time lapse imaging showing astrocyte mitochondrial dynamics}

\section{following astrocyte knockdown of miro.}

Representative time-lapse movie showing astrocyte mitochondria (R25H07-gal4, UASmito::GFP) dynamics in a miro knockdown brain $\left(U A S\right.$-miro $\left.{ }^{\mathrm{RNAi}}\right)$ at $8 \mathrm{~h}$ ALH. A single z-stack was acquired one a minute (each stack taking $15 "$ to acquire) and brains were imaged for a total of 10 minutes. Mitochondria were reduced in the neuropil.

\section{Supplementary Movie 11. Time lapse imaging showing astrocyte mitochondrial dynamics}

following astrocyte knockdown of $\mathrm{Dhc64C}$. 
1063 Representative time-lapse movie showing astrocyte mitochondria (R25H07-gal4, UAS-

1064 mito::GFP) dynamics in a Dhc64C knockdown brain $\left(U A S-D h c 64 C^{\mathrm{RNAi}}\right)$ at $8 \mathrm{~h}$ ALH. A single z-

1065 stack was acquired one a minute (each stack taking 15" to acquire) and brains were imaged for a

1066 total of 10 minutes. Mitochondria distributed normally in the neuropil. 